\title{
Solvability of rank one $p$-adic differential and $q$-difference equations over the Amice ring
}

\author{
Andrea Pulita
}

\begin{abstract}
We provide a necessary and sufficient condition for the solvability of a rank one differential (resp. $q$-difference) equation over the Amice's ring. We also extend to that ring a Birkoff decomposition result, originally due to Motzkin.
\end{abstract}

Version of May 31, 2021

\section{Contents}

INTRODUCTION 1

FIRST PART : SOLVABILITY OF RANK ONE DIFFERENTIAL EQUATIONS OVER $\mathcal{E}_{K} \quad 2$

1 Notations 2

1.1 Differential modules and radius of convergence $\ldots \ldots \ldots \ldots \ldots$

2 Criterion of solvability for differential equations over $\mathcal{E}_{K} \quad 5$

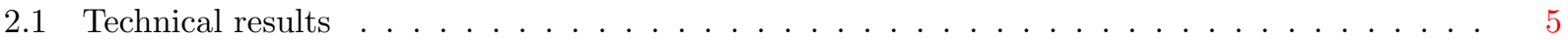

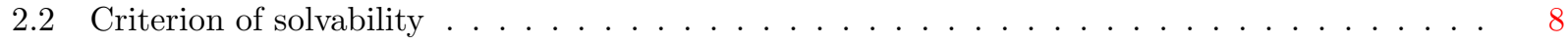

SECOND PART : SOLVABILITY OF RANK ONE $q$-DIFFERENCE EQUATIONS OVER $\mathcal{E}_{K}$

3 Some numerical Lemmas $\quad 11$

4 The Motzkin decomposition $\quad 13$

5 Criterion of solvability for $q$-difference equations over $\mathcal{E}_{K} \quad 15$

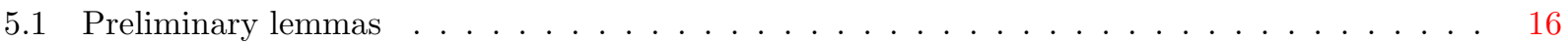

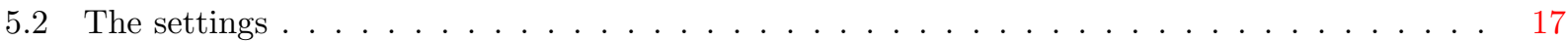

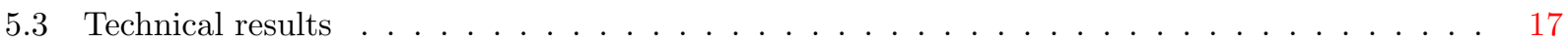

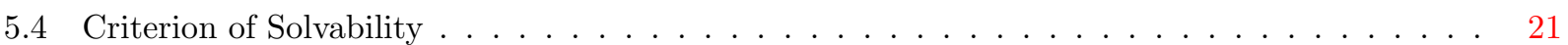

\section{INTRODUCTION}

Let $(K,||$.$) be a field of characteristic 0$ which is complete with respect to an ultramentric absolute value $|$.$| , and whose residual field k$ has positive characteristic $p>0$. Denote by $\mathscr{O}_{K}:=\{x \in$ $K|| x \mid \leqslant 1\}$ its ring of integers.

The Robba ring $\mathfrak{R}_{K}$ is the ring of power series $f(T)=\sum_{i \in \mathbb{Z}} a_{i} T^{i}, a_{i} \in K$, for which there exists an unspecified $\varepsilon<1$ (depending on $f$ ) such that $f(T)$ converges on the annulus $\{\varepsilon<|T|<1\}$. In a previous work [Pul07] (see also [CP09]) we described the isomorphism classes of rank one solvable differential equations over $\mathfrak{R}_{K}$. In particular we have obtained a criterion permitting to read in the coefficients of the differential equation the solvability.

In another work [Pul08] (see also [Pul14]) we studied the phenomena of deformation of $q$ difference equations and we have proved that, under the solvability condition, the category of dif- 


\section{AndRea Pulita}

ferential equation is equivalent to that of $q$-difference equations (this generalizes previous works of Yves André and Lucia Di Vizio [ADV04], [DV04]).

In this paper we are interested to differential and $q$-difference equations over the Amice's ring $\mathcal{E}_{K}$. This ring is formed by formal power series $f(T)=\sum_{i \in \mathbb{Z}} a_{i} T^{i}, a_{i} \in K$, that are bounded (i.e. $\left.\sup _{i}\left|a_{i}\right|<+\infty\right)$, and such that $\lim _{i \rightarrow-\infty}\left|a_{i}\right|=0$. It is the ring used by J.M.Fontaine in the theory of $(\phi, \Gamma)$-modules [Fon90].

A classification of rank one differential (or $q$-difference) equations over the ring $\mathcal{E}_{K}$ is not known, and it seems reasonable to think that such a classification will be quite different in nature with respect to that obtained in [Pul07] for differential equations over the Robba ring $\mathfrak{R}_{K}$. This will not be the goal of this paper. We here obtain a criterion of solvability for differential and $q$-difference equations similar to that in [Pul07].

We actually describe completely the precise nature of the solutions of differential and difference equations as exponentials of Artin-Hasse type.

As a corollary we obtain that every differential equation over $\mathcal{E}_{K}$ has a basis in which the associated operator has coefficients in $\mathscr{O}_{K}\left[\left[T^{-1}\right]\right]$. This constitutes an analogous of the Katz canonical extension theorem [Kat87] (see also [Mat02]).

The results of this paper have been obtained in 2005, during our PhD at the university of Paris, under the supervision of Gilles Christol.

Acknowledgments Step 4 in the proof of Proposition 2.1.1 is due to Gilles Christol, we want here to express our gratitude to him for helpful discussions.

\section{First PART : SOLVABILITY OF RANK ONE DIFFERENTIAL EQUATIONS OVER $\mathcal{E}_{K}$}

\section{Notations}

Let $\mathbb{R}_{\geqslant 0}$ be the interval of real numbers that are greater than or equal to 0 .

Let $K$ be a complete valued field of characteristic 0 , with ring of integers $\mathscr{O}_{K}:=\{x \in K,|x| \leqslant 1\}$, and maximal ideal $\mathfrak{p}_{K}:=\{x \in K,|x|<1\}$. We assume that the residual field $k:=\mathscr{O}_{K} / \mathfrak{p}_{K}$ has positive characteristic $p>0$.

If $I \subseteq \mathbb{R}_{\geqslant 0}$ is any interval, we denote by $\mathcal{A}_{K}(I)$ the ring of analytic functions on the space $\{|T| \in I\}$. If $0 \in I$ this is an open or closed disk, in this case we have

$$
\mathcal{A}_{K}(I):=\left\{\sum_{i \geqslant 0} a_{i} T^{i}, a_{i} \in K, \lim _{i \rightarrow \infty}\left|a_{i}\right| \rho^{i}=0, \text { for all } \rho \in I\right\} .
$$

If $0 \notin I$ it is an open, closed, or semi-open annulus and we have

$$
\mathcal{A}_{K}(I):=\left\{\sum_{i \in \mathbb{Z}} a_{i} T^{i}, a_{i} \in K, \lim _{i \rightarrow \pm \infty}\left|a_{i}\right| \rho^{i}=0, \text { for all } \rho \in I\right\} .
$$

For all $\rho \in I$ we have a norm on $\mathcal{A}_{K}(I)$ given by $\left|\sum_{i \in \mathbb{Z}} a_{i} T^{i}\right|_{\rho}:=\sup _{i}\left|a_{i}\right| \rho^{i}$. And $\mathcal{A}_{K}(I)$ is complete with respect to the Frechet topology defined by the family of norms $\left\{|\cdot|_{\rho}\right\}_{\rho \in I}$. We define the Robba ring as

$$
\mathfrak{R}_{K}:=\cup_{\varepsilon>0} \mathcal{A}_{K}(] 1-\varepsilon, 1[) .
$$

The topology of the ring $\mathfrak{R}_{K}$ is the limit of the topologies of $\mathcal{A}_{K}(] 1-\varepsilon, 1[)$ which are Frechet spaces. It is hence a $\mathcal{L F}$ topology. 
The Amice's ring $\mathcal{E}_{K}$ is defined as

$$
\mathcal{E}_{K}:=\left\{\sum_{i \in \mathbb{Z}} a_{i} T^{i}, a_{i} \in K, \sup _{i}\left|a_{i}\right|<+\infty, \lim _{i \rightarrow-\infty}\left|a_{i}\right|=0\right\} .
$$

It is a complete valued ring with respect to the Gauss norm $\left|\sum a_{i} T^{i}\right|_{1}:=\sup \left|a_{i}\right|$. Its ring of integers $\mathscr{O}_{\mathcal{E}_{K}}=\left\{\left.f \in \mathcal{E}_{K}|| f\right|_{1} \leqslant 1\right\}$ is a local ring, with residual field $k((t))$ (i.e. a field of Laurent power series with coefficients in $k$ ). If $K$ is discretely valued, $\mathcal{E}_{K}$ is moreover a field.

We define the bounded Robba ring as $\mathcal{E}_{K}^{\dagger}:=\mathfrak{R}_{K} \cap \mathcal{E}_{K}$. If $K$ is discretely valued, it is a field. $\mathfrak{R}_{K}$ and $\mathcal{E}_{K}$ induce two distinct topologies on $\mathcal{E}_{K}^{\dagger}$, and this last is dense in $\mathfrak{R}_{K}$ and in $\mathcal{E}_{K}$ with respect to the corresponding topologies.

\subsection{Differential modules and radius of convergence}

Let $A$ be one of the rings $\mathcal{A}_{K}(I)$ or $\mathcal{E}_{K}$. The $A$-module of continuous differentials $\Omega_{A / K}^{1}$ is free and one dimensional over $A$. Let $d: A \rightarrow A$ be a non trivial derivation corresponding to a generator of $\Omega_{A / K}^{1}$. A differential module over $A$ is a finite free $A$-module $M$, together with a linear map $\nabla: M \rightarrow M$, called connection, satisfying the Leibniz rule $\nabla(f m)=d(f) m+f \nabla(m), f \in A$, $m \in M$.

In this paper we will always assume the rank of $M$ to be 1 . We denote by $\partial_{T}:=T \frac{d}{d T}$. If a basis of $M$ is given, then $\nabla$ becomes an operator of the form $f \mapsto \partial_{T}(f)-g \cdot f: A \rightarrow A$, where $g \in A$. We say then that $M$ is defined by the operator $\partial_{T}-g$. With respect to another basis $M$ will be represented by another operator $\partial_{T}-g_{2}$, and $g_{2}$ is related to $g$ by the rule $g_{2}=g+\frac{\partial_{T}(h)}{h}$, where $h \in A^{\times}$is the base change matrix.

We denote by $M_{1} \otimes M_{2}$ the tensor product of two differential modules $\left(M_{1}, \nabla_{1}\right)$ and $\left(M_{2}, \nabla_{2}\right)$. This is a differential module whose underling $A$-module is $M_{1} \otimes_{A} M_{2}$, and whose connection is $\nabla_{1} \otimes \mathrm{Id}+\mathrm{Id} \otimes \nabla_{2}$. If $\partial_{T}-g_{1}$ and $\partial_{T}-g_{2}$ are associated operators with respect to some bases, then $\partial_{T}-\left(g_{1}+g_{2}\right)$ will be the operator of $M_{1} \otimes M_{2}$ with respect to the tensor product of the bases.

Let now $\partial_{T}-g(T)$ be a differential operator with $g \in \mathcal{A}_{K}(I)$, and let $\Omega / K$ be any complete valued field extension of $K$. For all $x \in \Omega,|x| \in I$, we look at $\Omega[[T-x]]$ as an $\mathcal{A}_{K}(I)$-differential algebra by the Taylor map

$$
f(T) \mapsto \sum_{k \geqslant 0}\left(\frac{d}{d T}\right)^{k}(f)(x) \frac{(T-x)^{k}}{k !}: \mathcal{A}_{K}(I) \longrightarrow \Omega[[T-x]] .
$$

Define inductively $g_{[k]}(T)$ as $g_{[0]}:=1, g_{[1]}:=g(T) / T$, and for all $k \geqslant 1$ we set $g_{[k+1]}:=\frac{d}{d T}\left(g_{[n]}\right)+$ $g_{[k]} g_{[1]}$. The Taylor solution of $\partial_{T}-g(T)$ at $x$ is then

$$
s_{x}(T):=\sum_{k \geqslant 0} g_{[k]}(x) \frac{(T-x)^{k}}{k !} .
$$

Indeed $\partial_{T}\left(s_{x}(T)\right)=g(T) s_{x}(T)$. The radius of convergence of $s_{x}(T)$ at $x$ is, by the usual definition,

$$
\liminf _{k}\left(\left|g_{[k]}(x)\right| /|k !|\right)^{-\frac{1}{k}} .
$$

Definition 1.1.1. We set

$$
\omega:=|p|^{\frac{1}{p-1}}<1 .
$$




\section{Andrea Pulita}

Definition 1.1.2. The radius of convergence of $M$ at $\rho \in I$ is

$$
\begin{aligned}
\operatorname{Ray}(M, \rho) & :=\min \left(\rho, \underset{k}{\liminf }\left(\left|g_{[k]}\right| \rho /|k !|\right)^{-1 / k}\right) \\
& =\min \left(\rho, \omega\left[\limsup _{k}\left(\left|g_{[k]}\right| \rho\right)^{1 / k}\right]^{-1}\right) .
\end{aligned}
$$

We say that $M$ is solvable at $\rho$ if $\operatorname{Ray}(M, \rho)=\rho$.

This number represents the minimum radius of convergence of a solution at an unspecified point $x$ of norm $|x|=\rho$. More precisely there exists a complete field extension $\Omega / K$ and a point $t_{\rho} \in \Omega$, with $\left|t_{\rho}\right|=\rho$, such that for all $g \in \mathcal{A}_{K}(I)$ one has $|g|_{\rho}=\left|g\left(t_{\rho}\right)\right|_{\Omega}$. Such a point is called a $\rho$-generic point (cf. [CR94]). We deduce that

$$
\operatorname{Ray}(M, \rho)=\min \left(\rho, \min _{|x|=\rho, x \in \Omega}\left\{\text { Radius of } s_{x}(T)\right\}\right) .
$$

Indeed this follows from (1.7) and from the fact that $\left|g_{[k]}\right|_{\rho}=\max _{|x|=\rho, x \in \Omega}\left|g_{[k]}(x)\right|_{\Omega}=\left|g_{[k]}\left(t_{\rho}\right)\right|$.

Remark 1.1.3. The second equality of (1.9) follows from the fact that the sequence $|k !|^{1 / k}$ is convergent to $\omega$, and $\left|g_{[k]}\right|_{\rho}^{1 / k}$ is bounded by $\max \left(\left|g_{[1]}\right|_{\rho}, \rho^{-1}\right)$. The presence of $\rho$ in the minimum makes this definition invariant under change of basis in $M$.

If now $\partial_{T}-g(T)$ is a differential operator with $g(T) \in \mathcal{E}_{K}$, then (1.9) has a meaning for $\rho=1$ and it is an invariant by base changes of $M$.

Remark 1.1.4. We shall recall the following facts, that will be systematically used in the sequel:

i) If $M$ is a differential module over $\mathcal{E}_{K}$, then Definition 1.1.2 has a meaning for $\rho=1$;

ii) If $M$ is a differential module over $\mathcal{A}_{K}(I)$, and if $I$ is not reduced to a point, then the function $\rho \mapsto \operatorname{Ray}(M, \rho)$ has the following properties

(a) It is continuous on $I$.

(b) It is piecewise of the form $\alpha \rho^{\beta}>0$, which is usually quoted as the log-affinity property (this means that the function $r \mapsto \log (\operatorname{Ray}(M, \exp (r)))=\log (\alpha)+\beta r$ is affine).

(c) The slopes $\beta$ are natural numbers.

iii) Recall that for all differential module $M, N$ one has

$$
\operatorname{Ray}(M \otimes N, \rho) \geqslant \min (\operatorname{Ray}(M, \rho), \operatorname{Ray}(N, \rho))
$$

and equality holds if $\operatorname{Ray}(M, \rho) \neq \operatorname{Ray}(N, \rho)$ (cf. [PulO\%, Remark 1.2]). Notice that if for a given $\rho$ we have $\operatorname{Ray}(M, \rho)=\operatorname{Ray}(N, \rho)$, it often happens that $\operatorname{Ray}\left(M, \rho^{\prime}\right) \neq \operatorname{Ray}\left(N, \rho^{\prime}\right)$ holds in a neighborhood of $\rho$ with the individual exception of $\rho$, so by continuity we deduce that (1.11) is an equality also at $\rho$.

iv) The $p$-th ramification $f(T) \mapsto f\left(T^{p}\right)$ is a $K$-linear ring endomorphism of $\mathcal{E}_{K}$ and of $\mathcal{A}_{K}(I)$ which is called (somehow improperly) Frobenius map. We denote it by $\varphi$. By extension of scalars one can define an exact endo-functor which is called pull-back by Frobenius, denoted by $\varphi^{*}$ (cf. [CM02], [Pul07, 1.2.3, 1.2.4]). The functor associates to a differential equation $\partial-g(T)$ the differential equation $\partial_{T}-p \cdot g\left(T^{p}\right)$. This is a technical tool of the theory used mainly to "move the radii" of convergence of a differential module. More precisely if $M$ is a differential module over $\mathcal{A}_{K}\left(I^{p}\right)$, then for all $\rho \in I$ one has

$$
\operatorname{Ray}\left(\varphi^{*}(M), \rho\right) \geqslant \rho \cdot \min \left(\left(\frac{\operatorname{Ray}\left(M, \rho^{p}\right)}{\rho^{p}}\right)^{1 / p},|p|^{-1} \frac{\operatorname{Ray}\left(M, \rho^{p}\right)}{\rho^{p}}\right),
$$

and equality holds if $\operatorname{Ray}\left(M, \rho^{p}\right) \neq \omega^{p} \rho^{p}$ (cf. [CM02, Thm.7.2], [Ked10, 10.3.2]). 
Solvability of RANK one $p$-ADIC Differential AND $q$-DIfFEREnCE EQUATIONS Over the Amice RING

If $\operatorname{Ray}\left(M, \rho^{p}\right)>\omega \rho^{p}$, it is known that the functor can be (improperly speaking) "inverted", this means that there exists a differential module $N$ such that $\varphi^{*}(N) \cong M$, and that such a module is unique (for a more precise statement see [CM02, Thm. 7.5], [Ked10, 10.4.2]). We say that $N$ is an antecedent by Frobenius of $M$.

We refer to [PulO'7], for the proof of these sentences and for all further properties and definitions.

\section{Criterion of solvability for differential equations over $\mathcal{E}_{K}$}

In this section we obtain a criterion of solvability for differential equations over $\mathcal{E}_{K}$. After a technical part (cf. Proposition 2.1.1), the main result will be actually an immediate consequence of the Lemma 2.2.1.

Lemma 2.0.5 (Small radius). Let $\partial_{T}-g(T), g(T) \in \mathcal{E}_{K}$. Then Ray $\left(\partial_{T}-g(T), 1\right)<\omega$ if and only if $|g(T)|_{1}>1$. In this case we have

$$
\operatorname{Ray}\left(\partial_{T}-g(T), 1\right)=\omega \cdot|g(T)|_{1}^{-1}
$$

Proof. See [Pul07, Lemma 1.1].

\subsection{Technical results}

There is no domain of the affine line where all the power series in $\mathcal{E}_{K}$ converge. If $\mathrm{M}$ is a differential module associated with the operator $\partial_{T}-g$, with $g \in \mathcal{E}_{K}$, it is useful to have a basis of M in which $g$ converges on some domain. For this, for all functions $g(T)=\sum_{i \in \mathbb{Z}} a_{i} T^{i}$ we set $g^{-}(T):=\sum_{i \leqslant-1} a_{i} T^{i}$, and $g^{+}(T):=\sum_{i \geqslant 1} a_{i} T^{i}$. The following proposition expresses any solvable $\mathrm{M}$ as tensor product of some solvable differential modules defined over a disk centered at 0 and a disk centered at $\infty$. The "Step 4" of the proof is due to G.Christol.

Proposition 2.1.1. Let $\partial_{T}-g(T), g(T) \in \mathcal{E}_{K}$, be an equation which is solvable at $\rho=1$. Then $\partial_{T}-g^{-}(T), \partial_{T}-a_{0}$, and $\partial_{T}-g^{+}(T)$ are all solvable at $\rho=1$.

Proof. - Step 1: By (1.6), the equation $\partial_{T}-g^{-}(T)$ (resp. $\partial_{T}-g^{+}(T)$ ) has a convergent solution at $\infty$ (resp. at 0$)$, hence $\operatorname{Ray}\left(\partial_{T}-g^{-}(T), \rho\right)=\rho$, for large values of $\rho\left(\operatorname{resp}\right.$. $\operatorname{Ray}\left(\partial_{T}-g^{-}(T), \rho\right)=\rho$, for $\rho$ close to 0$)$. On the other hand, a direct computation proves that there is a $R^{0}>0$ such that $\operatorname{Ray}\left(\partial_{T}-a_{0}, \rho\right)=R^{0} \cdot \rho$, for all $\rho$. Let

$$
\begin{aligned}
R^{-} & :=\operatorname{Ray}\left(\partial_{T}-g^{-}(T), 1\right), \\
R^{+} & :=\operatorname{Ray}\left(\partial_{T}-g^{+}(T), 1\right), \\
R^{0} & :=\operatorname{Ray}\left(\partial_{T}-a_{0}, 1\right) .
\end{aligned}
$$

We have to prove that $R_{0}=R^{-}=R^{+}=1$.

- Step 2: We begin by proving that $R^{+}=R^{-}$, and that $R^{0} \geqslant R^{-}=R^{+}$. In the following picture $R:=R^{-}=R^{+}$, and for all operators $L$, we let $r:=\log (\rho)$ and $R(L, r):=\log (\operatorname{Ray}(L, \rho) / \rho)$.

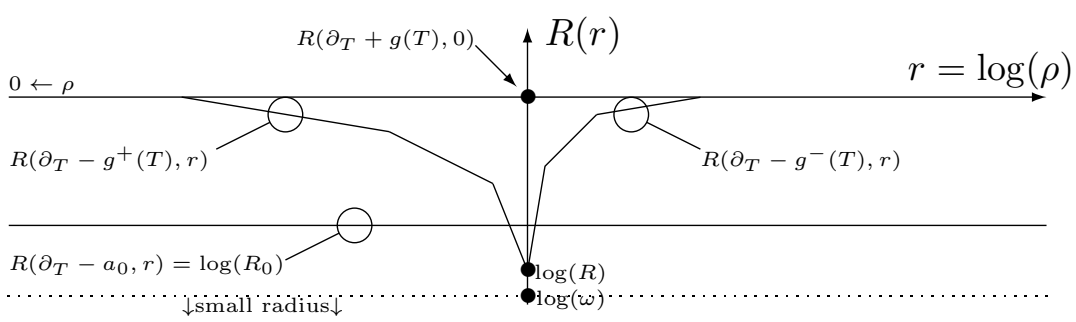




\section{Andrea Pulita}

Since $\partial_{T}-g$ is the tensor product of $\partial_{T}-g^{-}, \partial_{T}-g^{+}$, and $\partial_{T}-a_{0}$, we deduce from point iii) of Remark 1.1.4 that if two among $R^{-}, R^{+}, R^{0}$ are 1 , then the third is also equal to 1 .

Assume now by contrapositive that at least two among $R^{-}, R^{+}, R^{0}$ are strictly less than 1 . Then either $R^{-}<1$ or $R^{+}<1$. We want to prove that $R^{+}=R^{-}$, and that $R^{0} \geqslant R^{-}=R^{+}$.

We assume for instance that $R^{-}<1$, the case where $R^{+}<1$ can be proved symmetrically.

The function $r \mapsto R\left(\partial_{T}-g^{-}(T), r\right)$ is concave, and $\operatorname{Ray}\left(\partial_{T}-g^{-}(T), 1\right)=1$ if and only if the slope of $r \mapsto R\left(\partial_{T}-g^{-}(T), r\right)$ is 0 for $r \rightarrow 0^{+}$.

The map $r \mapsto R\left(\partial_{T}-g^{-}(T), r\right)$ for $r \rightarrow 0^{+}$is strictly positive and the slope of $R\left(\partial_{T}-a_{0}, r\right)=$ $\log \left(R_{0}\right)$ is 0 . We deduce from point iii) of Remark 1.1.4 that $\operatorname{Ray}\left(\partial_{T}-g^{-}(T), \rho\right) \neq \operatorname{Ray}\left(\partial_{T}-a_{0}, \rho\right)$ with the possible exception of an isolated $\rho$. Hence $\operatorname{Ray}\left(\partial_{T}-\left(a_{0}+g^{-}(T)\right), \rho\right)=\min \left(\operatorname{Ray}\left(\partial_{T}-\right.\right.$ $\left.\left.g^{-}(T), \rho\right), \rho R^{0}\right)$, for all $\rho>1$ close to 1 . By continuity, this equality holds at $\rho=1$, that is

$$
\operatorname{Ray}\left(\partial_{T}-\left(a_{0}+g^{-}(T)\right), 1\right)=\min \left(R^{-}, R^{0}\right) .
$$

Now since $\partial_{T}-g(T)$ is the tensor product of $\partial_{T}-g^{+}(T)$ and $\partial_{T}-\left(a_{0}+g^{-}(T)\right)$, and since $\operatorname{Ray}\left(\partial_{T}-g(T), 1\right)=1$, we have again by point iii) of Remark 1.1.4 that

$$
R^{+}:=\operatorname{Ray}\left(\partial_{T}-g^{+}(T), 1\right)=\operatorname{Ray}\left(\partial_{T}-\left(a_{0}+g^{-}(T)\right), 1\right)=\min \left(R^{-}, R^{0}\right) .
$$

We now claim that $R^{0} \geqslant R^{-}$, so the previous equality implies $R^{+}=R^{-}$. Indeed if $R^{-}>R^{0}$, then $R^{+}=R^{0}$. Hence, as above, by concavity we deduce that for all $\rho<1$ one has $\operatorname{Ray}\left(\partial_{T}-g^{+}(T), \rho\right) \neq$ $\operatorname{Ray}\left(\partial_{T}-a_{0}, \rho\right)$, and that $\operatorname{Ray}\left(\partial_{T}-\left(a_{0}+g^{+}(T)\right), 1\right)=R^{0}<R^{-}$. This implies $\operatorname{Ray}\left(\partial_{T}-g(T), 1\right)=$ $\min \left(R^{0}, R^{-}\right)=R^{0}<1$, contradicting the solvability of $\partial_{T}-g$. Hence we must have $R^{0} \geqslant R^{-}=R^{+}$.

-Step 3: If $R$ denotes the number $R^{-}=R^{+}$, then we have $R \geqslant \omega$. Indeed if $R^{-}<\omega$ or $R^{+}<\omega$, then, by 2.0.5, $\left|g^{-}(T)\right|_{1}>1$ or $\left|g^{+}(T)\right|_{1}>1$, hence $|g(T)|_{1}>1$ which is in contradiction with the small radius lemma 2.0.5, since the equation $\partial_{T}-g(T)$ is solvable.

- Step 4: We now prove that $R>\omega$. For this we need two lemmas:

Lemma 2.1.2 ([Chr83, 4.8.5]). Let $\partial_{T}-g(T), g(T) \in \mathcal{E}_{K},|g(T)|_{1} \leqslant 1$ be some equations. Then $\operatorname{Ray}\left(\partial_{T}-g(T), 1\right)>\omega$ if and only if $\left|g_{[s]}(T)\right|_{1}<1$, for some $s \geqslant 1{ }^{1}$

Lemma 2.1.3. If $\operatorname{Ray}\left(\partial_{T}-g(T), 1\right)>\omega$, where $g(T)=\sum a_{i} T^{i}$, then $\left|a_{i}\right|<1$, for all $i \leqslant-1$.

Proof. The matrix of $d / d T$ is $g_{[1]}:=g(T) / T$. By definition one has

$$
\begin{aligned}
\operatorname{Ray}\left(\partial_{T}-g(T), 1\right)=\operatorname{Ray}\left(d / d T-g_{[1]}(T), 1\right) & =\min \left(1, \liminf _{s}\left(\left|g_{[s]}(T)\right|_{1} /|s !|\right)^{-1 / s}\right) \\
& =\min \left(1, \omega \cdot \liminf _{s}\left(\left|g_{[s]}(T)\right|_{1}\right)^{-1 / s}\right),
\end{aligned}
$$

where $g_{[s]}(T)$ is associated to the derivation $\left(\frac{d}{d T}\right)^{s}$. Since $\operatorname{Ray}\left(\partial_{T}-g(T), 1\right)>\omega$, hence $\lim _{s \rightarrow \infty}\left|g_{[s]}(T)\right|_{1}=$ 0 . In particular $\left|g_{[s]}(T)\right|_{1}<1$, for some $s \geqslant 1$. Moreover, by the small radius lemma 2.0.5, we have $|g(T)|_{1} \leqslant 1$. We proceed by contrapositive: let $-d$ be the smallest index such that $\left|a_{-d}\right|=1$. The reduction of $g_{[1]}(T)=g(T) / T$ in $k((t))$ is of the form $\overline{g_{[1]}(T)}=\bar{a}_{-d} t^{-d-1}+\cdots$. If $-d \leqslant-1$, then an induction on the equation $g_{[s+1]}=\frac{d}{d x}\left(g_{[s]}\right)+g_{[s]} g_{[1]}$ shows that $\overline{g_{[s]}(T)}=\bar{a}_{-d}^{s} t^{(-d-1) s}+\cdots \neq 0$. This is in contradiction with the fact that $\left|g_{[s]}(T)\right|_{1}<1$, for some $s \geqslant 1$.

Let us show now that $R>\omega$. Since $R^{+}=R^{-}=R$, it is sufficient to show that $R^{-}>\omega$. By Lemma 2.1.3, we have $\left|a_{i}\right|<1$, for all $i \leqslant-1$. Since $\lim _{i \rightarrow-\infty}\left|a_{i}\right|=0$, hence $\left|g^{-}(T)\right|_{1}<1$. Then

${ }^{1}$ See Lemma 5.3 .5 for the q-analogue of this lemma. 
Lemma 2.1.2 implies $R^{-}>\omega$.

- Step 5: Since $R>\omega$, then we can take the antecedent by Frobenius of $\partial_{T}-g^{-}(T), \partial_{T}-g^{+}(T)$, $\partial_{T}-a_{0}$. More precisely, there exists $f^{+}(T)=\sum_{i \geqslant 0} b_{i}^{+} T^{i} \in \mathcal{A}\left(\left[0,1[)^{\times}, f^{-}(T)=\sum_{i \leqslant 0} b_{i}^{-} T^{i} \in\right.\right.$ $\mathcal{A}([1, \infty])^{\times}$, and there are functions $g^{(1),-}(T)=\sum_{i \leqslant 0} a_{i}^{(1),-} T^{i}, g^{(1),+}(T)=\sum_{i \geqslant 0} a_{i}^{(1),+} T^{i}, b_{0} \in K$ such that

$$
\begin{aligned}
p b_{0} & =a_{0}+n, \quad \text { for some } n \in \mathbb{Z}, \\
p g^{(1),-}\left(T^{p}\right)^{\sigma} & =g^{-}(T)+\frac{\partial_{T}\left(f^{-}(T)\right)}{f^{-}(T)}, \\
p g^{(1),+}\left(T^{p}\right)^{\sigma} & =g^{+}(T)+\frac{\partial_{T}\left(f^{+}(T)\right)}{f^{+}(T)},
\end{aligned}
$$

where $\sigma: K \rightarrow K$ is an endomorphism of fields lifting of the $p$-th power map of $k$, and $\left(\sum a_{i} T^{i}\right)^{\sigma}$ means $\sum \sigma\left(a_{i}\right) T^{i}$.

We see immediately that $b_{0}^{+} \neq 0$ and $b_{0}^{-} \neq 0$, and that $v_{T}\left(\partial_{T}\left(f^{+}\right) / f^{+}\right) \geqslant 1$ and $v_{T^{-1}}\left(\partial_{T}\left(f^{-}\right) / f^{-}\right) \geqslant$ 1 , where $v_{T}$ is the $T$-adic valuation, and $v_{T^{-1}}$ is the $T^{-1}$-adic valuation. Since $g^{-}(T)$ and $g^{+}(T)$ have no constant term, we deduce that $a_{0}^{(1),+}=0$ and $a_{0}^{(1),-}=0$. Observe now that both $f^{-}$and $f^{+}$belong to $\mathcal{E}_{K}^{\times}$, hence $\partial_{T}-\left(g^{(1),-}(T)+b_{0}+g^{(1),+}(T)\right)$ is an antecedent of Frobenius of $\partial_{T}-g(T)$, and it is then solvable.

- Step 6: Steps 1, 2, 3, 4 are still true for the antecedent. In particular, if we set

$$
\begin{aligned}
R^{-}(1) & :=\operatorname{Ray}\left(\partial_{T}-g^{(1),-}(T), 1\right), \\
R^{+}(1) & :=\operatorname{Ray}\left(\partial_{T}-g^{(1),+}(T), 1\right), \\
R^{0}(1) & :=\operatorname{Ray}\left(\partial_{T}-b_{0}, 1\right),
\end{aligned}
$$

then we must have $R^{-}(1)=R^{+}(1)>\omega$. Let $R(1):=R^{-}(1)=R^{+}(1)$, then $R(1)=R^{1 / p}$ by the property of the antecedent. This implies $R>\omega^{1 / p}$.

Now the condition $R(1)>\omega$, guarantee the existence of the antecedent of the antecedent, and the process can be iterated indefinitely. This shows that $R>\omega^{1 / p^{h}}$ for all $h \geqslant 0$, that is $R=1$.

Corollary 2.1.4. We have $a_{0} \in \mathbb{Z}_{p}$ and $\partial_{T}-g^{+}(T)$ is trivial.

Proof. By the transfer theorem, the Taylor solution at 0 of $\partial_{T}-g^{+}(T)$ is convergent in the open unit disk. This solution is invertible with inverse the solution of the dual differential module, hence it is bounded and belongs to $\mathcal{E}_{K}$.

The following corollary, together with Corollary 2.2.7, constitute the analogue of the Katz's canonical extension functor [Kat87]:

Corollary 2.1.5 (Katz's canonical extension). Let $M$ be a solvable rank one differential module over $\mathcal{E}_{K}$ represented in a basis by the operator $\partial_{T}-g(T)$, with $g(T)=\sum_{i \in \mathbb{Z}} a_{i} T^{i} \in \mathcal{E}_{K}$. Then there exists a basis of $M$ in which the associated operator is

$$
\partial_{T}-\left(a_{0}+g^{-}(T)\right) .
$$

In particular $M$ comes by scalar extension from a differential module over the closed unit disk $D:=\{|T| \geqslant 1\} \cup\{\infty\}$ centered at $\infty$. It has a regular singularity at $\infty$ if and only if $a_{0} \in \mathbb{Z}$, and it has no singularities on $D$ otherwise. 


\section{Andrea Pulita}

\subsection{Criterion of solvability}

Following [Pul07] we now introduce an exponential series which is the solution of our differential equations. We refer to [Pul07] for all notations and properties.

We set $\mathbb{J}:=\{n \in \mathbb{Z} \mid(n, p)=1, n \geqslant 1\}$.

For all ring $A$ (not necessarily with unit element) we denote by $\mathbf{W}(A)$ the ring of $p$-typical Witt vectors of infinite length with coefficients in $A$. Its elements are sequences $\boldsymbol{a}=\left(a_{0}, a_{1}, \ldots\right)$ of elements of $A$. For all $m \geqslant 0$ we call phantom vector of $\boldsymbol{a}$ the tuple $\phi_{m}(\boldsymbol{a}):=a_{0}^{p^{m}}+p a_{1}^{p^{m-1}}+\cdots+p^{m} a_{m}$. The map $\mathbf{W}(A) \rightarrow A^{\mathbb{N}}$ associating to $\boldsymbol{a}$ the tuple $\left(\phi_{0}(\boldsymbol{a}), \phi_{1}(\boldsymbol{a}), \ldots\right)$ is a morphism of functors in rings. In order to make a more evident distinction between Witt vectors and phantom components, we denote Witt vector by the letter $\lambda$ and phantom components by the letter $\phi$, moreover we also use a bracket $\left\langle\phi_{0}, \phi_{1}, \ldots\right\rangle$ to indicate an element of the Ring $A^{\mathbb{N}}$.

Let now $A=T \mathscr{O}_{K}[[T]]$. We now recall some notions from [Pul07, Section 4.3]. For all $\boldsymbol{\lambda}=$ $\left(\lambda_{0}, \lambda_{1}, \ldots\right) \in \mathbf{W}(K)$ and all integer $d>0$ we set

$$
\boldsymbol{\lambda} T^{d}:=\left(\lambda_{0} T^{d}, \lambda_{1} T^{p d}, \lambda_{2} T^{p^{2} d}, \ldots\right) \in \mathbf{W}(T K[[T]]) .
$$

To a sum $\sum_{d>0} \boldsymbol{\lambda}_{d} T^{d} \in \mathbf{W}(T K[[T]])$ we associate the following exponential of Artin-Hasse type

$$
E\left(\sum_{d>0} \boldsymbol{\lambda}_{d} T^{d}, 1\right)=\prod_{d>0} \exp \left(\sum_{m \geqslant 0} \phi_{d, m} \frac{T^{d p^{m}}}{p^{m}}\right)
$$

where for all $d>0$ the tuple $\left(\phi_{d, 0}, \phi_{d, 1}, \ldots\right)$ is the phantom vector of $\boldsymbol{\lambda}_{d}$. The map $\mathbf{W}(K) \rightarrow K^{\mathbb{N}}$ being an isomorphism, it easy to prove that any exponential of the form $\exp \left(\sum_{d>0} b_{d} \frac{T^{d}}{d}\right) \in 1+$ $T K[[T]]$ can be uniquely decomposed as

$$
\exp \left(\sum_{d>0} b_{d} \frac{T^{d}}{d}\right)=\exp \left(\sum_{n \in \mathbb{J}} \sum_{m \geqslant 0} b_{n p^{m}} \frac{T^{n p^{m}}}{n p^{m}}\right)=\exp \left(\sum_{n \in \mathbb{J}} \sum_{m \geqslant 0} \phi_{n, m} \frac{T^{n p^{m}}}{p^{m}}\right)=E\left(\sum_{n \in \mathbb{J}} \boldsymbol{\lambda}_{n} T^{n}, 1\right),
$$

where $\phi_{n, m}=b_{n p^{m}} / n$, and $\boldsymbol{\lambda}_{n} \in \mathbf{W}(K)$ is defined as the unique Witt vector with phantom vector $\left(\phi_{n, 0}, \phi_{n, 1}, \ldots\right)$. We refer to [Pul07, Section 4.3] for further properties.

The following Lemma asserts that solutions of rank one solvable differential equations over the open unit disk are those exponentials as above whose Witt vectors have coefficients in $\mathscr{O}_{K}$.

Lemma 2.2.1. The differential equation $\partial_{T}-g^{+}(T), g^{+}(T)=\sum_{i \geqslant 1} a_{i} T^{i} \in \mathcal{A}([0,1[)$ is solvable if and only if there exists a family $\left\{\boldsymbol{\lambda}_{n}\right\}_{n \in \mathbb{J}}, \boldsymbol{\lambda}_{n} \in \mathbf{W}\left(\mathscr{O}_{K}\right)$, with phantom components $\phi_{n}=$ $\left(\phi_{n, 0}, \phi_{n, 1}, \ldots\right)$ satisfying

$$
a_{n p^{m}}=n \phi_{n, m}, \quad \text { for all } n \in \mathbb{J}, m \geqslant 0 .
$$

In other words, we have $\exp \left(\sum_{i \geqslant 1} a_{i} \frac{T^{i}}{i}\right)=E\left(\sum_{n \in \mathbb{J}} \boldsymbol{\lambda}_{n} T^{n}, 1\right)$, where

$$
E\left(\sum_{n \in \mathbb{J}} \boldsymbol{\lambda}_{n} T^{n}, 1\right):=\exp \left(\sum_{n \in \mathbb{J}} \sum_{m \geqslant 0} \phi_{n, m} T^{n p^{m}} / p^{m}\right) .
$$

Proof. The formal series $E\left(\sum_{n \in \mathbb{J}} \boldsymbol{\lambda}_{n} T^{n}, 1\right) \in 1+T \mathscr{O}_{K}[[T]]$ is solution of the equation $L:=\partial_{T}-$ $\sum_{n \in \mathbb{J}} \sum_{m \geqslant 0} n \phi_{n, m} T^{n p^{m}}$. Since this exponential converges in the unit disk, then $\operatorname{Ray}(L, \rho)=\rho$, for all $\rho<1$, and $L$ is solvable.

Conversely, assume that $\partial_{T}-g^{+}(T)$ is solvable. Then the Witt vectors $\boldsymbol{\lambda}_{n}=\left(\lambda_{n, 0}, \lambda_{n, 1}, \ldots\right)$ are defined by the relation (2.14). For example, for all $n \in \mathbb{J}$ we have

$$
\lambda_{n, 0}=\frac{a_{n}}{n}, \quad \lambda_{n, 1}=\frac{1}{p}\left(\frac{a_{n p}}{n}-\left(\frac{a_{n}}{n}\right)^{p}\right) .
$$


We must show that $\left|\lambda_{n, m}\right| \leqslant 1$, for all $n \in \mathbb{J}, m \geqslant 0$.

- Step 1: By the small radius Lemma 2.0.5, we have $\left|a_{i}\right| \leqslant 1$, for all $i \geqslant 1$. Hence, by (2.16), for all $n \in \mathbb{J}$, we have $\left|\lambda_{n, 0}\right| \leqslant 1$. Then the exponential

$$
E\left(\sum_{n \in \mathbb{J}}\left(\lambda_{n, 0}, 0,0, \ldots\right) T^{n}, 1\right)=\exp \left(\sum_{n \in \mathbb{J}} \sum_{m \geqslant 0} \lambda_{n, 0}^{p^{m}} \frac{T^{n p^{m}}}{p^{m}}\right)
$$

converges in the unit disk and is solution of the operator $Q^{(0)}:=\partial_{T}-h^{(0)}(T)$, where $h^{(0)}(T)=$ $\sum_{n \in \mathbb{J}} \sum_{m \geqslant 0} n \lambda_{n, 0}^{p^{m}} T^{n p^{m}}$. So $Q^{(0)}$ is solvable.

- Step 2: The tensor product operator $\partial_{T}-\left(g^{+}(T)-h^{(0)}(T)\right)$ is again solvable and satisfies $g^{+}(T)-h^{(0)}(T)=p \cdot g^{(1)}\left(T^{p}\right)$, for some $g^{(1)}(T) \in T K[[T]]$. In other words, the "antecedent by ramification" $\varphi_{p}^{*}$ of the equation $\partial_{T}-\left(g^{+}(T)-h^{(0)}(T)\right)$ is given by $\partial_{T}-g^{(1)}(T)$, which is then solvable.

- Step 3: We observe that $g^{(1)}(T)=\frac{1}{p} \sum_{n \in \mathbb{J}} \sum_{m \geqslant 0}\left(a_{n p^{m+1}}-n\left(\frac{a_{n}}{n}\right)^{p^{m+1}}\right) T^{n p^{m}}$, and again by the small radius lemma, we have $\left|a_{n p}-n\left(\frac{a_{n}}{n}\right)^{p}\right| \leqslant|p|$, which implies $\left|\lambda_{n, 1}\right| \leqslant 1$.

The process can be iterated indefinitely. This proves that $\left|\lambda_{n, m}\right| \leqslant 1$ for all $n, m$.

Remark 2.2.2. We shall now consider the general case of an equation $\partial_{T}-g(T)$, with $g(T)=$ $\sum_{i \in \mathbb{Z}} a_{i} T^{i} \in \mathcal{E}_{K}$, and get a criterion of solvability. Suppose that $\partial_{T}-g(T)$ is solvable. We know that $\partial_{T}-g^{-}(T), \partial_{T}-a_{0}$ and $\partial_{T}-g^{+}(T)$ are all solvable (cf. 2.1.1). We can then consider $\partial_{T}-g^{-}(T)$ as an operator on $] 1, \infty]$ (instead of $[1, \infty]$ ), and the precedent lemma 2.2.1 give us the existence of a family of Witt vector $\left\{\boldsymbol{\lambda}_{-n}\right\}_{n \in \mathbb{J}} \subset \mathbf{W}\left(\mathscr{O}_{K}\right)$, satisfying $a_{-n p^{m}}=-n \phi_{-n, m}$, for all $n \in \mathbb{J}$, and all $m \geqslant 0$. Conversely, suppose given two families $\left\{\boldsymbol{\lambda}_{-n}\right\}_{n \in \mathbb{J}}$ and $\left\{\boldsymbol{\lambda}_{n}\right\}_{n \in \mathbb{J}}$, with $\boldsymbol{\lambda}_{n} \in \mathbf{W}\left(\mathscr{O}_{K}\right)$. Since the phantom components of $\boldsymbol{\lambda}_{n}$ are bounded by 1 , then $\left|a_{i}\right|$ is bounded by 1 , and then $g^{+}(T)$ belongs to $\mathcal{E}_{K}$.

What we need now is a condition on the family $\left\{\boldsymbol{\lambda}_{-n}\right\}_{n \in \mathbb{J}}$ in order that the series

$$
g^{-}(T):=\sum_{n \in \mathbb{J}} \sum_{m \geqslant 0}-n \phi_{-n, m} T^{-n p^{m}}
$$

belongs to $\mathcal{E}_{K}$.

Proposition 2.2.3. Let $\left\{\boldsymbol{\lambda}_{-n}\right\}_{n \in J}, \boldsymbol{\lambda}_{-n} \in \mathbf{W}\left(\mathscr{O}_{K}\right)$, be a family of Witt vectors. Let $\left\langle\phi_{-n, 0}, \phi_{-n, 1}, \ldots\right\rangle$ be the phantom vector of $\boldsymbol{\lambda}_{-n}:=\left(\lambda_{-n, 0}, \lambda_{-n, 1}, \ldots\right)$. The series

$$
g^{-}(T):=\sum_{n \in \mathbb{J}} \sum_{m \geqslant 0}-n \phi_{-n, m} T^{-n p^{m}},
$$

belongs to $\mathcal{E}_{K}$ if and only if

$$
\left\{\begin{aligned}
\left|\lambda_{-n, m}\right|<1, & \text { for all } n \in \mathbb{J}, \text { for all } m \geqslant 0 ; \\
\lim _{n \in \mathbb{J}, n \rightarrow \infty} \lambda_{-n, m}=0 \quad, \quad \text { for all } m \geqslant 0, &
\end{aligned}\right.
$$

as in the picture

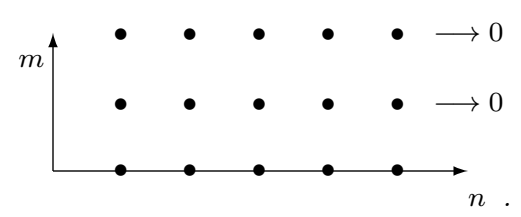

We need the following lemma: 


\section{Andrea Pulita}

Lemma 2.2.4. Let $\boldsymbol{\lambda}=\left(\lambda_{0}, \lambda_{1}, \ldots\right) \in \mathbf{W}\left(\mathscr{O}_{K}\right)$ be a Witt vector, and let $\left\langle\phi_{0}, \phi_{1}, \ldots\right\rangle \in \mathscr{O}_{K}^{\mathbb{N}}$ be its phantom vector. Then $\phi_{j} \rightarrow 0$ in $\mathscr{O}_{K}$ if and only if $\left|\lambda_{j}\right|<1$, for all $j \geqslant 0$.

Proof. The set of Witt vectors whose phantom components go to 0 is clearly an ideal $I \subset \mathbf{W}\left(\mathscr{O}_{K}\right)$ containing $\mathbf{W}\left(\mathfrak{p}_{K}\right)$, where $\mathfrak{p}_{K}$ is the maximal ideal of $\mathscr{O}_{K}$. Reciprocally, suppose $\phi_{j} \rightarrow 0$, since $\phi_{j}=\lambda_{0}^{p^{j}}+p \lambda_{1}^{p^{j-1}}+\cdots+p^{j} \lambda_{j}$, we have $\left|\lambda_{0}\right|<1$. Then $\boldsymbol{\lambda}^{(1)}:=\left(0, \lambda_{1}, \lambda_{2}, \ldots\right)=\boldsymbol{\lambda}-\left(\lambda_{0}, 0, \ldots\right)$ lies again in the ideal $I$, and hence $\phi_{j}\left(\boldsymbol{\lambda}^{(1)}\right)=p \lambda_{1}^{p^{j-1}}+p^{2} \lambda_{2}^{p^{j-2}}+\cdots+p^{j} \lambda_{j} \rightarrow 0$. This shows that $\left|\lambda_{1}\right|<1$. Proceeding inductively one sees that $\left|\lambda_{j}\right|<1$, for all $j \geqslant 0$.

We now are ready to give the proof of Proposition 2.2.3 :

Proof. Assume that $g^{-}(T)=\sum_{n \in \mathbb{J}} \sum_{m \geqslant 0}-n \phi_{-n, m} T^{-n p^{m}}$ lies in $\mathcal{E}_{K}$. This happens if and only if $\lim _{n p^{m} \rightarrow \infty} \phi_{-n, m}=0$, and implies $\lim _{m \rightarrow \infty} \phi_{-n, m}=0$ for all $n \in \mathbb{J}$. By Lemma 2.2.4, we have $\left|\lambda_{-n, m}\right|<1$, for all $n \in \mathbb{J}$ and all $m \geqslant 0$. An easy induction shows that $\lim _{n \in \mathbb{J}, n \rightarrow \infty} \lambda_{-n, m}=0$, for all $m \geqslant 0$.

Reciprocally, assume that $\left\{\boldsymbol{\lambda}_{-n}\right\}_{n \in \mathbb{J}}$ satisfies the condition (2.18). We must show that $\lim _{n p^{m} \rightarrow \infty} \phi_{-n, m}=$ 0 . For all $\varepsilon>0$, we choose $k \geqslant 0$ such that $\left|p^{k+1}\right|<\varepsilon$. By assumption, for all $0 \leqslant m \leqslant k$, there exists $N_{m}$ such that $\left|\lambda_{-n, m}\right|<\varepsilon$, for all $n \geqslant N_{m}$. Let $N:=\max \left(N_{0}, \ldots, N_{k}\right)$. Then

$$
\phi_{-n, m}=\underbrace{\lambda_{-n, 0}^{p^{m}}+\cdots+p^{k} \lambda_{-n, k}^{p^{m-k}}}_{<\varepsilon, \text { if } n \geqslant N}+\underbrace{p^{k+1} \lambda_{-n, k+1}^{p^{m-k-1}}+\cdots+p^{m} \lambda_{-n, m}}_{<\varepsilon} .
$$

Hence $\left|\phi_{-n, m}\right|<\varepsilon$, if $n \geqslant N$. On the other hand, by assumption, there is $\delta<1$ such that $\left|\lambda_{-n, m}\right| \leqslant$ $\delta<1$, for all $m=0, \ldots, k, n=0, \ldots, N$. Hence there exists $M$ such that $\left|\lambda_{-n, 0}^{p^{m}}\right|, \ldots,\left|\lambda_{-n, k}^{p^{m-k}}\right|<\varepsilon$, for all $m \geqslant M$. Then $\left|\phi_{-n, m}\right| \leqslant \varepsilon$, for all $n \geqslant N, m \geqslant M$. Hence $\lim _{n p^{m} \rightarrow \infty} \phi_{-n, m}=0$.

Definition 2.2.5. We denote by $\operatorname{Conv}(\mathcal{E})$ the set of families $\left\{\boldsymbol{\lambda}_{-n}\right\}_{n \in \mathbb{J}}$, with $\boldsymbol{\lambda}_{-n}=\left(\lambda_{-n, 0}, \lambda_{-n, 1}, \ldots\right) \in$ $\mathbf{W}\left(\mathscr{O}_{K}\right)$, satisfying condition (2.18).

Corollary 2.2.6 (Criterion of solvability). The equation $\partial_{T}-g(T), g(T)=\sum_{i \in \mathbb{Z}} a_{i} T^{i} \in \mathcal{E}_{K}$, is solvable if and only if $a_{0} \in \mathbb{Z}_{p}$, and there exist two families $\left\{\boldsymbol{\lambda}_{-n}\right\}_{n \in \mathbb{J}}$ and $\left\{\boldsymbol{\lambda}_{-n}\right\}_{n \in \mathbb{J}}$ with $\boldsymbol{\lambda}_{-n}, \boldsymbol{\lambda}_{n} \in$ $\mathbf{W}\left(\mathscr{O}_{K}\right)$, for all $n \in \mathbb{J}$, such that $\left\{\boldsymbol{\lambda}_{-n}\right\}_{n \in \mathbb{J}} \in \operatorname{Conv}\left(\mathcal{E}_{K}\right)$, and moreover

$$
a_{n p^{m}}=n \phi_{n, m} \quad, \quad a_{-n p^{m}}=-n \phi_{-n, m} .
$$

In other words, its formal solution $T^{a_{0}} \exp \left(\sum_{i \leqslant-1} a_{i} \frac{T^{i}}{i}\right) \exp \left(\sum_{i \geqslant 1} a_{i} \frac{T^{i}}{i}\right)$ can be represented by the symbol

$$
T^{a_{0}} \cdot \exp \left(\sum_{n \in \mathbb{J}} \sum_{m \geqslant 0} \phi_{-n, m} \frac{T^{-n p^{m}}}{p^{m}}\right) \cdot \exp \left(\sum_{n \in \mathbb{J}} \sum_{m \geqslant 0} \phi_{n, m} \frac{T^{n p^{m}}}{p^{m}}\right),
$$

where $\left(\phi_{-n, 0}, \phi_{-n, 1}, \ldots\right)$ (resp. $\left.\left(\phi_{n, 0}, \phi_{n, 1}, \ldots\right)\right)$ is the phantom vector of $\boldsymbol{\lambda}_{-n}$ (resp. $\left.\boldsymbol{\lambda}_{n}\right)$, and hence

$$
g(T)=\sum_{n \in \mathbb{J}} \sum_{m \geqslant 0}-n \phi_{-n, m} T^{-n p^{m}}+a_{0}+\sum_{n \in \mathbb{J}} \sum_{m \geqslant 0} n \phi_{n, m} T^{n p^{m}} .
$$

Corollary 2.2.7 (Katz's canonical extension). Let $\partial_{T}-\operatorname{Mod}\left(\mathcal{A}_{K}([1, \infty])\right)_{r k=1}^{\text {sol }}$ be the category of rank one differential modules over $\mathcal{A}_{K}([1, \infty])$, solvable at all $\rho \geqslant 1$, with a regular singularity at $\infty$ (i.e. the matrix of $\partial_{T}$ converge at $\infty$ and hence belongs to $\left.\mathcal{A}_{K}([1, \infty])\right)$. The scalar extension functor

$$
\partial_{T}-\operatorname{Mod}\left(\mathcal{A}_{K}([1, \infty])\right)_{r k=1}^{\mathrm{sol}} \longrightarrow \partial_{T}-\operatorname{Mod}\left(\mathcal{E}_{K}\right)_{r k=1}^{\text {sol }}
$$

is an equivalence. 
Solvability of RANK one $p$-ADIC Differential And $q$-DifFerence EqUations over the Amice RING

Proof. Corollary 2.2.6 shows that gives a correspondence between the objects. Indeed, all differential equations $\partial_{T}-g(T)$ over $g(T)=g^{-}(T)+a_{0}+g^{+}(T) \in \mathcal{E}_{K}$ is isomorphic over $\mathcal{E}_{K}$ to the equation $\partial_{T}-\left(g^{-}(T)+a_{0}\right)$. On the other hand, let $M, N \in \partial_{T}-\operatorname{Mod}\left(\mathcal{A}_{K}([1, \infty])\right)_{r k=1}^{\text {sol }}$, and let $\partial_{T}-g_{M}$, $\partial_{T}-g_{N}$ be the operators corresponding to a chosen basis of $M$ and $N$. An element of $\operatorname{Hom}(M, N) \stackrel{\sim}{\rightarrow}$ $M^{\vee} \otimes N$ is then a solution of the operator $\partial_{T}-\left(g_{N}-g_{M}\right)$. This solution will be of the form $y(T)=T^{a_{0}} \exp \left(\sum_{n \in \mathbb{J}} \sum_{m \geqslant 0} \phi_{-n, m} T^{-n p^{m}} / p^{m}\right)$, for some $\phi$. Since we are supposing that this solution belongs to $\mathcal{A}_{K}([1, \infty])$, then $a_{0} \in \mathbb{Z}$ and this exponential lies in $\mathcal{A}_{K}([1, \infty])$. Since the same argument works for $\operatorname{Hom}_{\partial_{T}}\left(M \otimes \mathcal{E}_{K}, N \otimes \mathcal{E}_{K}\right)$, and since $\mathcal{A}_{K}([1, \infty]) \subset \mathcal{E}_{K}$, then the map $\operatorname{Hom}_{\partial_{T}}(M, N) \rightarrow$ $\operatorname{Hom}_{\partial_{T}}\left(M \otimes \mathcal{E}_{K}, N \otimes \mathcal{E}_{K}\right)$ is bijective.

Remark 2.2.8. We are not able to obtain a complete description of the isomorphism class of a given differential equation over $\mathcal{E}_{K}$. Namely, over the Robba ring $\mathfrak{\Re}_{K}$, we know that a solution of a differential equation lies in $\mathfrak{R}_{K}$ if and only if the corresponding Witt vector (in a convenient basis of $M$ ) satisfies a certain property [PulO7, Theorem 3.1]. But we do not have the analogous result over $\mathcal{E}_{K}$. In other words, we do not have a necessary and sufficient condition on the Witt vector $\sum_{n \in \mathbb{J}} \boldsymbol{\lambda}_{-n} T^{-n}$ in order that $E\left(\sum_{n \in \mathbb{J}} \boldsymbol{\lambda}_{-n} T^{-n}, 1\right)$ belongs to $\mathcal{E}_{K}$.

\section{SECOND PART : SOLVABILITY OF RANK ONE $q$-DIFFERENCE EQUATIONS OVER $\mathcal{E}_{K}$}

We shall establish the $q$-analogue of the results of section 2. In order to do that, we will need some numerical lemmas (cf. section 3) and a generalization of the result of E.Motzkin (cf. [Mot77], and section 4 below). As a consequence we will prove that for $|q-1|<\omega$ we have an equivalence of $q$-confluence as in [Pul08].

We shall point out that, almost all statements are true for $|q-1|<1$. The only obstructions to obtain the confluence in the case $\omega \leqslant|q-1|<1$ are

i) the existence of the "antecedent by Frobenius" (used in "Step 5" of Proposition 5.3.2), which is proved in [DV04] only for $|q-1|<\omega$;

ii) the "Step 0" of Theorem 5.4.1.

Namely, the existence of an antecedent by Frobenius holds with $|q-1|<1$ over the Robba Ring, but the proof uses the Confluence [Pul08]. It is reasonable to conjecture that a more direct proof is possible generalizing [DV04] to the case $|q-1|<1$. The author hopes that these difficulties will be overcoming in future.

For these reasons the hypothesis $|q-1|<\omega$ will be introduced systematically starting from 5.3.6 on. Before Hypothesis 5.3.6 we will suppose that $|q-1|<1$.

\section{Some numerical Lemmas}

Lemma 3.0.9. Let us fix an integer $j \geqslant 0$. If $j \geqslant 1$ we assume $\omega^{1 / p^{j-1}}<\rho<\omega^{1 / p^{j}}$, and if $j=0$ we assume $\rho<\omega$. Then

$$
\frac{\rho^{p^{j}}}{\left|p^{j}\right|}>\sup \left(\rho^{r} /|r|: r \geqslant 1, r \neq p^{j}\right)
$$

Moreover, we have

$$
\rho<\frac{\rho^{p}}{|p|}<\cdots<\frac{\rho^{p^{j-1}}}{\left|p^{j-1}\right|}<\frac{\rho^{p^{j}}}{\left|p^{j}\right|} \quad ; \quad \frac{\rho^{p^{j}}}{\left|p^{j}\right|}>\frac{\rho^{p^{j+1}}}{\left|p^{j+1}\right|}>\frac{\rho^{p^{j+2}}}{\left|p^{j+2}\right|}>\cdots
$$

Proof. If $r \neq p^{k}$, for all $k \geqslant 0$, then $|r|=|p|^{v}$, with $v:=v_{p}(r)$, hence $\rho^{r} /|r|<\rho^{p^{v}} /|p|^{v}$. This 
proves (3.1). Now the condition $\rho^{p^{k-1}} /\left|p^{k-1}\right|<\rho^{p^{k}} /\left|p^{k}\right|$ is equivalent to $\rho_{1}<\frac{\rho_{1}^{p}}{|p|}$, where $\rho_{1}:=\rho^{p^{k-1}}$, and it is verified if and only if $\rho_{1}>\omega$, that is $\rho>\omega^{\frac{1}{p^{k-1}}}$. On the other hand, the inequality $\rho^{p^{k-1}} /\left|p^{k-1}\right|>\rho^{p^{k}} /\left|p^{k}\right|$ is equivalent to $\rho<\omega^{\frac{1}{p^{k}}}$.

Lemma 3.0.10. Let $n \geqslant 1$ be a natural number. Let $l(n):=\left[\log _{p}(n)\right]$, where $[x]$ denotes the greatest integer smaller than or equal to the real number $x$. Then for all $k \geqslant n$ we have

$$
\left|\frac{k !}{n !}\right|^{\frac{1}{k-n}} \geqslant|p|^{l(n)+1} .
$$

In particular, if $c \leqslant|p|^{l(n)+1}$, then for all $k \geqslant n$ we have

$$
\frac{c^{n}}{|n !|} \geqslant \frac{c^{k}}{|k !|} \text {. }
$$

Proof. If $k=n$, the relation is trivial; suppose $k>n$. The equation (3.4) is equivalent to $c \leqslant\left|\frac{k !}{n !}\right| \frac{1}{k-n}$. Since $|n !|=\omega^{n-S_{n}}$, where $S_{n}$ is the sum of the digits of the base $p$ expansion of $n$, then $\left|\frac{k !}{n !}\right|^{\frac{1}{k-n}}=$ $\omega^{1+\frac{S_{n}-S_{k}}{k-n}}$. If $n=n_{0}+n_{1} p+n_{2} p^{2}+\cdots+n_{l(n)} p^{l(n)}$, with $0 \leqslant n_{i} \leqslant p-1$, then $S_{n}=n_{0}+n_{1}+\cdots+n_{l(n)}$, hence $1 \leqslant S_{n} \leqslant(p-1)(l(n)+1)$. This shows that

$$
1+\frac{S_{n}-S_{k}}{k-n} \leqslant 1+\frac{(p-1)(l(n)+1)-1}{k-n} \leqslant 1+(p-1)(l(n)+1)-1=(p-1)(l(n)+1) .
$$

Hence $\left|\frac{k !}{n !}\right|^{\frac{1}{k-n}} \geqslant \omega^{(p-1)(l(n)+1)}=|p|^{l(n)+1}$, for all $k>n$.

Definition 3.0.11. Let $q \in K$ be such that $|q-1|<1$. For all complete valued field extension $\Omega / K$, and all $\alpha \in \Omega$ we define

$$
q^{\alpha}:=((q-1)+1)^{\alpha}:=\sum_{k \geqslant 0}\left(\begin{array}{l}
\alpha \\
k
\end{array}\right)(q-1)^{k},
$$

where $\left(\begin{array}{l}\alpha \\ k\end{array}\right):=\frac{\alpha(\alpha-1)(\alpha-2) \cdots(\alpha-k+1)}{k !}$.

If $|\alpha|>1$, then $\left|\left(\begin{array}{c}\alpha \\ k\end{array}\right)\right|=\frac{|\alpha|^{k}}{|k !|}$, hence $q^{\alpha}$ converges exactly for $|q-1|<\omega /|\alpha|$.

If $|\alpha| \leqslant 1$, then $q^{\alpha}$ converges at least for $|q-1|<\omega$, in particular if $\alpha \in \mathbb{Z}_{p}$, then $q^{\alpha}$ converges at least for $|q-1|<1$. For a detailed discussion on the radius of convergence of $q^{\alpha}$ see [DGS94, Ch.IV, Prop.7.3].

Lemma 3.0.12. Let $\alpha \in \Omega$ and $q \in K$ be as in Definition 3.0.11. Then

$$
\lim _{q \rightarrow 1} \frac{q^{\alpha}-1}{q-1}=\alpha
$$

Proof. Write $\frac{\left(q^{\alpha}-1\right)}{(q-1)}=\frac{((q-1)+1)^{\alpha}-1}{(q-1)}=\alpha+\sum_{k \geqslant 2}\left(\begin{array}{l}\alpha \\ k\end{array}\right)(q-1)^{k-1}$. Let $s:=\max (|\alpha|, 1)$, and for all $n \geqslant 1$ let $l(n):=\left[\log _{p}(n)\right]$. We now prove that if $|q-1| \leqslant|p|^{l(2)+1} / s$, then for all $k \geqslant 2$ we have $\left|\left(\begin{array}{l}\alpha \\ k\end{array}\right)(q-1)^{k-1}\right| \leqslant\left|\left(\begin{array}{l}\alpha \\ 2\end{array}\right)(q-1)\right|$ which is enough to conclude.

Assume $k \geqslant n \geqslant 1$. The condition $\left|\left(\begin{array}{l}\alpha \\ k\end{array}\right)(q-1)^{k-1}\right| \leqslant\left|\left(\begin{array}{l}\alpha \\ n\end{array}\right)(q-1)^{n-1}\right|$ is equivalent to

$$
|q-1| \leqslant\left|\left(\begin{array}{l}
\alpha \\
n
\end{array}\right) /\left(\begin{array}{l}
\alpha \\
k
\end{array}\right)\right|^{\frac{1}{k-n}}=\left(\frac{|k !|}{|n !|} \frac{1}{|(\alpha-n) \cdots(\alpha-k+1)|}\right)^{\frac{1}{k-n}} .
$$

By Lemma 3.0.10 we know that $\left(\frac{k !}{n !}\right)^{\frac{1}{k-n}} \geqslant|p|^{l(n)+1}$. On the other hand, it is clear that $\mid(\alpha-$ 
$n) \cdots(\alpha-k+1) \mid \leqslant s^{k-n}$. Hence the right hand side of (3.8) is bigger than $|p|^{l(n)+1} / s$. The claim is proved.

Lemma 3.0.13. Let $j \geqslant 0$. If $j=0$, assume that $|q-1|<\omega$, and if $j \geqslant 1$ we assume that $\omega^{1 / p^{j-1}}<|q-1|<\omega^{1 / p^{j}}$. Let $d:=\alpha p^{m} \in \mathbb{Z}_{p}$, with $\alpha \in \mathbb{Z}_{p}$ such that $(\alpha, p)=1$. Let $i:=\min (m, j)$. Then

$$
\left|q^{d}-1\right|=|d| \cdot \frac{|q-1|^{p^{i}}}{|p|^{i}}=\left|p^{m-i}\right||q-1|^{p^{i}}
$$

Proof. Since $(\alpha, p)=1$, hence $\left|\left(\begin{array}{c}\alpha \\ 1\end{array}\right)\right|=1$. Then

$$
\left|q^{\alpha}-1\right|=\left|((q-1)+1)^{\alpha}-1\right|=\left|\sum_{k=1}^{\infty}\left(\begin{array}{l}
\alpha \\
k
\end{array}\right)(q-1)^{k}\right|=|q-1| .
$$

Moreover, one has $\left|q^{\alpha p^{m}}-1\right|=\left|\left(\left(q^{\alpha}-1\right)+1\right)^{p^{m}}-1\right|=\left|\sum_{k=1}^{p^{m}}\left(\begin{array}{c}p^{m} \\ k\end{array}\right)\left(q^{\alpha}-1\right)^{k}\right|$. Since for all $k \leqslant p^{m}$ one has $\left|\left(\begin{array}{c}p^{m} \\ k\end{array}\right)\right|=\frac{|p|^{m}}{|k|}$, we deduce $\left|\left(\begin{array}{c}p^{m} \\ k\end{array}\right)\left(q^{\alpha}-1\right)^{k}\right|=\left|p^{m}\right| \frac{\rho^{k}}{|k|}$. The claim follows from Lemma 3.0.9 applied to $\rho=|q-1|=\left|q^{\alpha}-1\right|$.

\section{The Motzkin decomposition}

In [Mot77] a decomposition theorem for analytic element over an affinoïd domain of the line (i.e. a set of type $\left.\mathbb{P}_{K}^{1}-\cup_{i=1, \ldots, n} \mathrm{D}_{K}^{-}\left(a_{i}, r_{i}\right)\right)$ is proved. In [Chr81] G.Christol generalizes this decomposition for matrices with coefficients in analytic functions. We now generalize that theorem for series in $\mathcal{E}_{K}$ (cf. 4.0.17).

Let $I \subseteq \mathbb{R}_{\geqslant 0}$ be any non empty interval. We set $I_{0}:=I \cup[0, \rho]$ (resp. $I_{\infty}:=I \cup[\rho,+\infty]$ ), where $\rho \in I$. As an example if $I=\left[r_{1}, r_{2}\left[\right.\right.$ then $I_{0}=\left[0, r_{2}\left[\right.\right.$ and $I_{\infty}=\left[r_{1},+\infty\right]$.

Theorem 4.0.14. Let $I \subseteq \mathbb{R}_{\geqslant 0}$ be any interval. Then each invertible function $a(T) \in \mathcal{A}_{K}(I)^{\times}$can be uniquely written as

$$
a(T)=\lambda \cdot T^{N} \cdot a^{-}(T) \cdot a^{+}(T),
$$

where $\lambda \in K, N \in \mathbb{Z}, a^{+}(T)=1+\alpha_{1} T+\alpha_{2} T^{2}+\cdots \in 1+T \mathcal{A}_{K}\left(I_{0}\right)^{\times}$and $a^{-}(T)=1+\alpha_{-1} T^{-1}+$ $\alpha_{-2} T^{-1}+\cdots \in 1+T^{-1} \mathcal{A}_{K}\left(I_{\infty}\right)^{\times}$.

Before giving the proof we need two lemmas. Let $\bar{I}$ be the closure of $I$ in $\mathbb{R}$. Invertible functions are bounded, so it has a meaning to consider their norm $|.|_{\rho}$ for all $\rho \in \bar{I}$.

Lemma 4.0.15. Let $a^{+}(T)=1+\alpha_{1} T+\alpha_{2} T^{2}+\cdots$ be an invertible function in $\mathcal{A}_{K}\left(I_{0}\right)$. If $r \in I_{0}$, for all $i \geqslant 1$ we have $\left|\alpha_{i}\right| r^{i}<1$. If $r \in \overline{I_{0}}$ for all $i \geqslant 1$ we have $\left|\alpha_{i}\right| r^{i} \leqslant 1$.

The same claim holds for functions $a^{-}(T) \in \mathcal{A}_{K}\left(I_{\infty}\right)$.

Proof. By replacing $T$ with $\gamma_{r} T$, with $\left|\gamma_{r}\right|=r$, we can suppose $r=1$.

Since $a^{+}$is invertible, its valuation polygon has no breaks (cf. [CR94, Chapitre 2]), so for all $\rho \leqslant 1$ we have $\left|a^{+}\right|_{\rho}=\left|a^{+}(0)\right|=1$. Hence $\left|\alpha_{i}\right| \leqslant 1$ for all $i \geqslant 1$.

If now $r=1 \in I_{0}$, and if there exists $i \geqslant 1$ such that $\left|\alpha_{i}\right|=1$, the reduced series $\overline{a^{+}(T)} \in k[T]$ is a non constant polynomial. The zeros of $\overline{a^{+}(T)}$ lift into zeros of $a^{+}(T)$, which contradicts the fact that $a^{+}(T)$ is invertible, hence without zeros in the closed unit disks.

Lemma 4.0.16. Let $\rho \in \bar{I}$. Let $a^{-}(T)=1+\alpha_{-1} T^{-1}+\alpha_{-2} T^{-2}+\cdots \in \mathcal{A}_{K}\left(I_{\infty}\right)^{\times}$, and $a^{+}(T)=$ 


\section{Andrea Pulita}

$1+\alpha_{1} T+\alpha_{2} T^{2}+\cdots \in \mathcal{A}_{K}\left(I_{0}\right)^{\times}$be invertible functions. Then

$$
\left|a^{-}(T) \cdot a^{+}(T)-1\right|_{\rho}<1 .
$$

Proof. Write $a^{-}(T) a^{+}(T)=\sum_{n \in \mathbb{Z}} c_{n} T^{n}$. If we define $\alpha_{0}:=1$, then, for all $n \geqslant 0$ one has $c_{n}=\sum_{k=0}^{\infty} \alpha_{n+k} \alpha_{-k}$, and $c_{-n}=\sum_{k=0}^{\infty} \alpha_{-n-k} \alpha_{k}$. By Lemma 4.0.15, either for all $k \geqslant 1$ we have $\left|\alpha_{-k}\right| \rho^{-k}<1$, and $\left|\alpha_{k}\right| \rho^{k} \leqslant 1$, or for all $k \geqslant 1$ we have $\left|\alpha_{-k}\right| \rho^{-k} \leqslant 1$, and $\left|\alpha_{k}\right| \rho^{k}<1$. Since $\lim _{k \rightarrow \pm \infty}\left|\alpha_{k}\right| \rho^{k}=0$ then for all $n \geqslant 1$ one have $\left|c_{n}\right| \rho^{n}<1$, and $\left|c_{-n}\right| \rho^{-n}<1$, and $\left|c_{0}-1\right|<1$.

Proof of Theorem 4.0.14. We first prove the claim for a rational fraction $a=P / Q, P, Q \in K[T]$. Let $Z_{0}$ and $V_{0}$ (resp. $Z_{\infty}$ and $V_{\infty}$ ) be the set of its zeros and poles respectively whose valuation belongs to $I_{0}\left(\right.$ resp. $\left.I_{\infty}\right)$. Since $\operatorname{Gal}\left(K^{\text {alg }} / K\right)$ acts by isometric maps, the polynomials $P_{0}:=\prod_{z \in Z_{0}-\{0\}}(T-$ $z), P_{\infty}:=\prod_{z \in Z_{\infty}}(T-z), Q_{0}:=\prod_{v \in V_{0}-\{0\}}(T-v), Q_{\infty}:=\prod_{v \in V_{\infty}}(T-v)$ lie in $K[T]$ since their coefficients are invariant by Galois. Now $P=\alpha T^{s} P_{0} P_{\infty}$ and $Q=\beta T^{r} Q_{0} Q_{\infty}$, for convenient $\alpha, \beta \in K, r, s \in \mathbb{N}$. We then have $a^{+}(T)=\alpha^{\prime} P_{\infty} / Q_{\infty}, a^{-}(T)=\beta^{\prime} P_{0} / Q_{0}$, for convenient constants $\alpha^{\prime}, \beta^{\prime} \in K$.

We now deduce by density the case where $I$ is a compact interval. If $\|\cdot\|_{I}$ is the sup-norm on $\{|T| \in I\}$, the Frechet topology of $\mathcal{A}_{K}(I)$ is given by the individual norm $\|\cdot\|_{I}$, and $\left(\mathcal{A}_{K}(I),\|\cdot\|_{I}\right)$ is a Banach algebra.

Let $a(T)=\sum_{i \in \mathbb{Z}} b_{i} T^{i}$ be as in the claim. For all $\rho \in I$ we have $\lim _{i \rightarrow \pm \infty}\left|b_{i}\right| \rho^{i}=0$ so for all $\rho \in I$ we can consider the integer $N_{\rho}:=\min \left(\left.i|| b_{i}\left|\rho^{i}=\right| a(T)\right|_{\rho}\right)$. Since $a$ is invertible, the log-function $r \mapsto \log \left(|a(T)|_{\exp (r)}\right)$ is affine on $\bar{I}$ of slope $N \in \mathbb{Z}$. This means that $N_{\rho}=N$ for all $\rho \in \bar{I}-\inf (I)$. Moreover if $\inf (I) \in I$ the equality also holds at $\rho=\inf (I)$ by [CR94, Thm. 5.4.7]. Multiplying by $\left(b_{N} T^{N}\right)^{-1}$ we can assume $N=0$ and $|a|_{\rho}=1$ for all $\rho \in \bar{I}$.

Let $a_{n}(T)$ be a sequence of rational fractions convergent to $a(T)$. Then for $n$ sufficiently large $a_{n}(T)$ has no poles nor zeros on $\{|T| \in I\}$, hence $a_{n}(T)$ admits such a decomposition: $a_{n}(T)=$ $\lambda_{n} T^{N_{n}} a_{n}^{-}(T) a_{n}^{+}(T)$. Moreover there exists $n_{0}$ such that for all $n \geqslant n_{0}$ we have $N_{n}=0$, and $\left|\lambda_{n}\right|=1$.

We now prove that, if $a_{n}^{+}=1+h_{n}^{+}$and $a_{n}^{-}=1+h_{n}^{-}$, then for all $n, m \geqslant n_{0}$ the norms $\left|\lambda_{n}-\lambda_{m}\right|$, $\left\|a_{n}^{+}-a_{m}^{+}\right\|_{I}=\left\|h_{n}^{+}-h_{m}^{+}\right\|_{I}$, and $\left\|a_{n}^{-}-a_{m}^{-}\right\|_{I}=\left\|h_{n}^{-}-h_{m}^{-}\right\|_{I}$ are all bounded by $\left\|a_{n}-a_{m}\right\|_{I}$. Since $T^{-1} \mathcal{A}_{K}\left(\left[r_{1}, \infty\right]\right)$ and $T \mathcal{A}_{K}\left(\left[0, r_{2}\right]\right)$ are closed sets in $\mathcal{A}_{K}(I)$, this will be enough to show that the sequences $n \mapsto \lambda_{n}, n \mapsto h_{n}^{-}$, and $n \mapsto h_{n}^{+}$, all converge in $K, T^{-1} \mathcal{A}_{K}\left(\left[r_{1}, \infty\right]\right)$ and $T \mathcal{A}_{K}\left(\left[0, r_{2}\right]\right)$ respectively. This will be enough to obtain the desired decomposition (4.1).

Let $n, m \geqslant n_{0}$. We let $1+h^{-}:=\frac{1+h_{n}^{-}}{1+h_{m}^{-}}$and $1+h^{+}:=\frac{1+h_{m}^{+}}{1+h_{n}^{+}}$. Then

$$
\begin{aligned}
\left\|a_{n}-a_{m}\right\|_{I}=\left\|\lambda_{n} a_{n}^{-} a_{n}^{+}-\lambda_{m} a_{m}^{-} a_{m}^{+}\right\|_{I} & =\left\|\frac{\lambda_{n} a_{n}^{-} a_{n}^{+}-\lambda_{m} a_{m}^{-} a_{m}^{+}}{a_{n}^{+} a_{m}^{-}}\right\|_{I} \\
& =\left\|\lambda_{n} \frac{a_{n}^{-}}{a_{m}^{-}}-\lambda_{m} \frac{a_{m}^{+}}{a_{n}^{+}}\right\|_{I} \\
& =\left\|\left(\lambda_{n}-\lambda_{m}\right)+\lambda_{n} h^{-}-\lambda_{m} h^{+}\right\|_{I}
\end{aligned}
$$

We now notice that $h^{-}$(resp. $h^{+}$) is a power series of the form $b_{-1} T^{-1}+b_{-2} T^{-2}+\cdots$ (resp. $\left.b_{1} T+b_{2} T^{2}+\cdots\right)$, hence for all $\rho \in I$ we have

$$
\begin{aligned}
\left|\left(\lambda_{n}-\lambda_{m}\right)+\lambda_{n} h^{-}-\lambda_{m} h^{+}\right|_{\rho} & =\max \left(\left|\lambda_{n}-\lambda_{m}\right|,\left|\lambda_{n}\right| \sup _{i \leqslant-1}\left|b_{i}\right| \rho^{i},\left|\lambda_{m}\right| \sup _{i \geqslant 1}\left|b_{i}\right| \rho^{i}\right) \\
& =\max \left(\left|\lambda_{n}-\lambda_{m}\right|,\left|h^{-}\right|_{\rho},\left|h^{+}\right|_{\rho}\right) .
\end{aligned}
$$

So we find

$$
\left\|a_{n}-a_{m}\right\|_{I}=\sup \left(\left|\lambda_{n}-\lambda_{m}\right|,\left\|h^{-}\right\|_{I},\left\|h^{+}\right\|_{I}\right)
$$




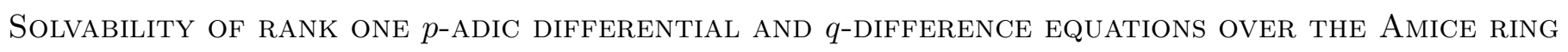

Now $\left\|h^{+}\right\|_{I}=\left\|\frac{1+h_{m}^{+}}{1-h_{n}^{+}}-1\right\|_{I}=\left\|\frac{h_{m}^{+}-h_{n}^{+}}{1-h_{n}^{+}}\right\|_{I}=\left\|h_{m}^{+}-h_{n}^{+}\right\|_{I}$, and analogously $\left\|h^{-}\right\|_{I}=\left\|h_{m}^{-}-h_{n}^{-}\right\|_{I}$. This gives the desired inequalities.

The case where $I$ is non compact is deduced by expressing $I$ as increasing union of compact intervals $J_{n} \subset J_{n+1} \subset I$. The uniqueness of the decomposition shows that the decomposition over $J_{n}$ coincides with that over $J_{n+1}$, and we conclude.

Theorem 4.0.17. Assume that $K$ is discretely valuated. Let $a(T) \in \mathcal{E}_{K}$. Then there exist $\lambda \in K$, $N \in \mathbb{Z}, a^{-}(T)=1+h^{-}(T)$ invertible in $1+T^{-1} \mathcal{A}_{K}([1, \infty])$, with $h^{-}(T)=\sum_{i \leqslant-1} \alpha_{i} T^{i}$, and $a^{+}(T)=1+h^{+}(T)$ invertible in $1+T \mathcal{A}_{K}\left(\left[0,1[)\right.\right.$, with $h^{+}(T)=\sum_{i \geqslant 1} \alpha_{i} T^{i}$, such that

$$
a(T)=\lambda \cdot T^{N} \cdot a^{-}(T) \cdot a^{+}(T) .
$$

Moreover, such a decomposition is unique.

Proof. The claim can not be deduced immediately "by density" because rational fractions are not dense in $\mathcal{E}_{K}$ with respect to the Gauss norm |.| $\left.\right|_{1}$. However the claim holds for functions in $\mathcal{E}_{K}^{\dagger}$ because they converge on some annulus. ${ }^{2}$ Now $\mathcal{E}_{K}^{\dagger}$ is dense in $\mathcal{E}_{K}$ with respect to the Gauss norm.

The assumption $K$ discretely valued arises now to prove that $\inf \left\{i \in \mathbb{Z}\right.$, such that $\left.\left|b_{i}\right|=|a(T)|_{1}\right\}$ is not equal to $+\infty$. This guarantee the existence of $N<+\infty$. We can now reproduce the same proof as Theorem 4.0.14 replacing $\|.\|_{I}$ by the Gauss norm $|\cdot|_{1}$. We obtain the desired decomposition.

Remark 4.0.18. As already mentioned, if the functions converge in some appropriate domains, the above results extend to matrices [Chr81], [CM02, Thm.6.5]. We do not know whether such a generalization exists for matrices with coefficients in $\mathcal{E}_{K}$. The main applications from our point of view would be the study of differential equations with coefficients in that ring.

\section{Criterion of solvability for $q$-difference equations over $\mathcal{E}_{K}$}

Hypothesis 5.0.19. From now on the valuation on $K$ will be discrete valuation, in order to have theorem 4.0.17.

We denote by

$$
\begin{array}{rlrl}
\sigma_{q}: f(T) \mapsto f(q T), & \partial_{T} & :=T \frac{d}{d T}, \\
d_{q}:=\frac{\sigma_{q}-1}{(q-1) T}, & \Delta_{q}:=\frac{\sigma_{q}-1}{q-1} .
\end{array}
$$

Let $A$ be one of the rings $\mathfrak{R}_{K}, \mathcal{E}_{K}, \mathcal{E}_{K}^{\dagger}, \mathcal{A}_{K}(I)$. A $q$-difference equation is finite free $A$-module $M$ together with an automorphism $\sigma_{q}: M \stackrel{\sim}{\rightarrow} M$ satisfying $\sigma_{q}(a m)=\sigma_{q}(a) \sigma_{q}(m)$ for all $a \in A$, $m \in M$. This corresponds in a basis of $M$ to an expression of the form $\sigma_{q}(Y)=a(q, T) Y$, where $a(q, T) \in G L_{n}(A)$.

From the action of $\sigma_{q}: M \stackrel{\sim}{\rightarrow} M$ we can define the action of $d_{q}$ and $\Delta_{q}$ on $M$. In a basis of $M$ the action of $d_{q}$ amounts to an equation of the form $d_{q}(Y)=g_{[1]}(q, T) Y$, with $g_{[1]}(q, T)=\frac{a(q, T)-\mathrm{Id}}{q-1} \in$ $M_{n}(A)$. As for differential equations we can attribute to such a module a radius of convergence. Namely the formal solution is given by

$$
Y_{q}(T, t):=\sum_{s \geqslant 0} g_{[s]}(q, t) \cdot \frac{(T-t)_{q, s}}{[s]_{q}^{!}}
$$

\footnotetext{
${ }^{2}$ Actually rational fractions are dense in $\mathcal{E}_{K}^{\dagger}$ with respect to the $\mathcal{L F}$ topology induced by the Robba ring $\mathfrak{R}_{K}$.
} 


\section{Andrea Pulita}

where for all natural $n \geqslant 0$

$$
[n]_{q}^{!}:=\frac{\prod_{i=1}^{n}\left(q^{i}-1\right)}{(q-1)^{n}}
$$

is the $q$-factorial, and $(T-t)_{q, s}:=(T-t)(T-q t) \cdots\left(T-q^{s-1} t\right)$ (cf. [Pul08] for more details), and

$g_{[s]}$ is the matrix of the action of $d_{q}^{n}$ on $M$. Namely $g_{[0]}=\mathrm{Id}, g_{[1]}=\frac{a(q, T)-1}{(q-1) T}$, and for all $s \geqslant 2$ one has $g_{[s+1]}(q, T)=d_{q}\left(g_{[s]}(q, T)\right)+\sigma_{q}\left(g_{[s]}(q, T)\right) \cdot g_{[1]}(q, T)$.

The radius of convergence of $d_{q}-g_{[1]}$ is then defined as

$$
\operatorname{Ray}\left(d_{q}-g_{[1]}(q, T), \rho\right):=\min \left(\liminf _{s}\left(\left|g_{[s]}(q, T)\right|_{\rho} /[s]_{q}^{!}\right)^{-1 / s}, \rho\right)
$$

This number is attached to the operator $d_{q}-g_{[1]}$, but it is not invariant by base changes of $M$. The radius is always less than or equal to $\rho$, if it is equal to $\rho$ we say that $\sigma_{q}-a(q, T)$ is solvable at $\rho$. If $A=\mathcal{E}_{K}$ and $\rho=1$ we simply say solvable (without specifying $\rho=1$ ). If $A=\mathfrak{R}_{K}$, we say that the equation is solvable if $\lim _{\rho \rightarrow 1^{-}} \operatorname{Ray}\left(\sigma_{q}-a(q, T), \rho\right)=1$.

\subsection{Preliminary lemmas}

Lemma 5.1.1. Assume $|q-1|<1$. Then the sequence $\left|[n]_{q}^{!}\right|^{1 / n}$ converges to a real number strictly less than 1 , we call $\omega_{q}<1$ that number. Moreover, let $\kappa$ be the smallest integer such that $\left|q^{\kappa}-1\right|<\omega$, then

$$
\omega_{q}=\left\{\begin{array}{ccc}
\omega & \text { if } & \kappa=1 \\
\left(\left|\frac{q^{\kappa}-1}{q-1}\right| \cdot \omega\right)^{\frac{1}{\kappa}} & \text { if } & \kappa \geqslant 2 .
\end{array}\right.
$$

Proof. [DV04, 3.5].

Lemma 5.1.2. Let $|q-1|<1$. For all $f(T) \in \mathcal{A}_{K}(I)$, for all $\rho \in I$ and all $k \geqslant 1$, we have $\left|\frac{d_{q}^{k}}{[k]_{q}^{!}}(f)\right|_{\rho} \leqslant \rho^{-k}|f|_{\rho}$. The same result is true for $f \in \mathcal{E}_{K}$ and $\rho=1$.

Proof. [DV04, 2.1].

Lemma 5.1.3 ( $q$-Small Radius, $q$-analogue of Lemma 2.0.5). Let $q \in K,|q-1|<1$, and let $I \subseteq \mathbb{R}_{\geqslant 0}$ be any interval. Let $\sigma_{q}-a(q, T)$, a $(q, T) \in \mathcal{A}_{K}(I)$ be some rank one q-difference equation. Let $R_{\rho}:=\operatorname{Ray}\left(\sigma_{q}-a(q, T), \rho\right)$ be the radius of convergence of the equation at $\rho \in I$. Then

$$
R_{\rho} \geqslant \frac{\omega_{q}}{\max \left(\left|g_{[1]}(q, T)\right|_{\rho}, \rho^{-1}\right)}=\frac{\omega_{q} \cdot \rho \cdot|q-1|}{\max \left(|a(q, T)-1|_{\rho},|q-1|\right)}
$$

Moreover $R_{\rho}<\omega_{q} \cdot \rho$ if and only if $|a(q, T)-1|_{\rho}>|q-1|$, and in this case

$$
R_{\rho}=\frac{\omega_{q} \cdot \rho \cdot|q-1|}{|a(q, T)-1|_{\rho}} .
$$

The same assertions hold for solvable q-difference equations over $\mathcal{E}_{K}$, with $\rho=1$.

Proof. Let $g_{[s]}(q, T) \in \mathcal{A}_{K}(I)$ be the $1 \times 1$ matrix of $\left(d_{q}\right)^{s}$. By definition

$$
\begin{aligned}
\operatorname{Ray}\left(d_{q}-g_{[1]}(q, T), 1\right) & =\min \left(\rho, \liminf _{s}\left(\left|g_{[s]}(q, T)\right|_{1} /\left|[s]_{q}^{!}\right|\right)^{-\frac{1}{s}}\right) \\
& =\min \left(\rho, \omega_{q} \cdot \liminf _{s}\left(\left|g_{[s]}(q, T)\right|_{1}\right)^{-\frac{1}{s}}\right) .
\end{aligned}
$$

One has inductively $\left|g_{[s]}\right|_{\rho} \leqslant \max \left(\left|g_{[1]}\right|_{\rho}, \rho^{-1}\right)^{s}$, this shows (5.6). Moreover, if $\left|g_{[1]}\right|_{\rho}>\rho^{-1}$, then $\left|g_{[s]}\right|_{\rho}=\max \left(\left|g_{[1]}\right|_{\rho}, \rho^{-1}\right)^{s}$ and (5.7) holds. Reciprocally, if $R_{\rho}<\omega_{q} \cdot \rho$, then, by (5.6), one has $|a(q, T)-1|>|q-1|$. 
Solvability of RANK one $p$-ADIC Differential And $q$-DifFerence EqUations over the Amice RING

Lemma 5.1.4. Let $|q-1|<1$. Let $\sigma_{q}-a(q, T)$ be a rank one solvable equation such that $a(q, T) \in$ $\mathfrak{R}_{K}$ or $a(q, T) \in \mathcal{E}_{K}$. Let $a(q, T)=\lambda_{q} T^{N} a^{-}(q, T) a^{+}(q, T)$ be the Motzkin decomposition of $a(q, T)$ (cf. Theorems 4.0.14, 4.0.17), then $N=0$ and $\left|\lambda_{q}-1\right|<1$.

Proof. The solvability implies $|a(q, T)-1|_{1} \leqslant|q-1|<1$ (cf. Lemma 5.1.3), hence $|a(q, T)|_{1}=1$. More precisely, with the notations as in the proof of Lemma 4.0.16, one has $\left|\lambda_{q} \sum_{n \in \mathbb{Z}} c_{n} T^{n+N}-1\right|_{1} \leqslant$ $|q-1|<1$. We know that $\sup _{n \neq 0}\left|c_{n}\right|<1$ and $\left|c_{0}-1\right|<1$ (cf. Lemma 4.0.16). If $N \neq 0$, then $\left|\lambda_{q} c_{0} T^{N}\right|_{1}<1$ and $\left|\lambda_{q} c_{-N}-1\right|<1$. The first one implies $\left|\lambda_{q}\right|<1$, which contradicts the second one. Hence $N=0$. We deduce that $\left|\lambda_{q} c_{0}-1\right|<1$ which implies $\left|\lambda_{q}-1\right|<1$.

Lemma 5.1.5. Let $|q-1|<1$. There exists $R_{0}>0$ such that Ray $\left(\sigma_{q}-\lambda_{q}, \rho\right)=R_{0} \cdot \rho$, for all $\rho \in[0, \infty[$.

Proof. By [DV04, 1.2.4], one has

$$
\left|g_{[n]}(T)\right|_{\rho}^{\frac{1}{n}}=\frac{\left|\sum_{j=0}^{n}(-1)^{j}\left(\begin{array}{c}
n \\
j
\end{array}\right)_{q^{-1}} q^{\frac{-j(j-1)}{2}} \lambda_{q}^{j}\right|^{1 / n}}{|q-1| \cdot \rho} .
$$

Since the numerator does not depend on $\rho$, the lemma is proved.

\subsection{The settings}

As for differential equations, we shall find a description of the formal solution of a given solvable $q$-difference equation

$$
\sigma_{q}\left(y_{q}\right)=a(q, T) \cdot y_{q},
$$

with $a(q, T) \in \mathcal{E}_{K}$. We will show that solutions of $q$-difference equations are actually solutions of differential equation of the form (2.20). By Lemma 5.1.4, we know that

$$
a(q, T)=\lambda_{q} \cdot a^{-}(q, T) \cdot a^{+}(q, T),
$$

with $a^{-}(q, T):=1+\sum_{i \leqslant-1} \alpha_{i} T^{i}$, and $a^{+}(q, T):=1+\sum_{i \geqslant 1} \alpha_{i} T^{i}$. Now write formally

$$
a^{-}(q, T):=\exp \left(\sum_{i \leqslant-1} a_{i} T^{i}\right), \quad a^{+}(q, T):=\exp \left(\sum_{i \geqslant 1} a_{i} T^{i}\right) .
$$

Then the formal solution of (5.9) is

$$
y_{q}(T):=\exp \left(\sum_{i \leqslant-1} \frac{a_{i}}{q^{i}-1} T^{i}\right) \cdot q^{a_{0}} \cdot \exp \left(\sum_{i \geqslant 1} \frac{a_{i}}{q^{i}-1} T^{i}\right) .
$$

We are interested to study this exponential in the case in which the equation (5.9) is solvable. The main result will be the Criterion of solvability 5.4.6.

\subsection{Technical results}

In this section $q \in \mathrm{D}^{-}(1,1)$ is fixed. We will omit the index $q$ in the series. The following proposition is the q-analogue of Proposition 2.1.1 for the Robba ring.

Proposition 5.3.1. Let $|q-1|<1$. Let $\sigma_{q}-a(T), a(T)=\lambda a^{-}(T) a^{+}(T) \in \mathfrak{R}_{K}$ be a solvable equation. Then $\sigma_{q}-a^{-}(T), \sigma_{q}-\lambda, \sigma_{q}-a^{+}(T)$ are all solvable.

Proof. With analogous notations of Proposition 2.1.1, we find the following picture: 
Andrea Pulita

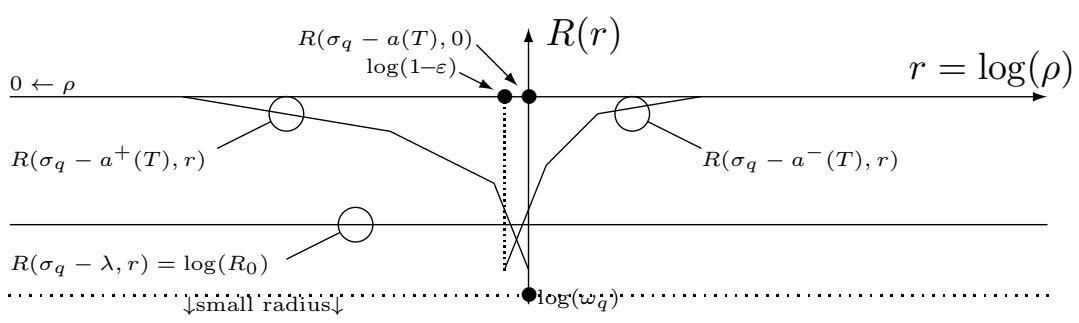

Since there exists a common interval $I:=] 1-\varepsilon, 1$ [ in which all operators exist, and since the slope of $\operatorname{Ray}\left(\sigma_{q}-a^{-}, \rho\right)$ (resp. $\operatorname{Ray}\left(\sigma_{q}-a^{+}, \rho\right)$ ) is strictly positive (resp. negative) in $I$, hence there are at most 3 points in which these graphics cross. Hence, by continuity, for all $\rho \in I$ one has

$$
\operatorname{Ray}\left(\sigma_{q}-a, \rho\right)=\min \left(\operatorname{Ray}\left(\sigma_{q}-a^{-}, \rho\right), \operatorname{Ray}\left(\sigma_{q}-a^{+}, \rho\right), \operatorname{Ray}\left(\sigma_{q}-\lambda, \rho\right)\right) .
$$

By assumption $\lim _{\rho \rightarrow 1^{-}} \operatorname{Ray}\left(\sigma_{q}-a, \rho\right)=1$, hence the claim follows.

We now give the q-analogue of Proposition 2.1.1 for the ring $\mathcal{E}_{K}$ :

Proposition 5.3.2. Let $|q-1|<\omega$. Let $\sigma_{q}-a(T), a(T)=\lambda a^{-}(T) a^{+}(T) \in \mathcal{E}_{K}$, be a solvable equation. Then $\sigma_{q}-a^{-}(T), \sigma_{q}-\lambda, \sigma_{q}-a^{+}(T)$ are all solvable.

Proof. Steps 1 and 2 of this proof coincide with the same steps of the proof of Proposition 2.1.1. We will expose it without proofs for fixing notation. The first part of this proposition does not use the hypothesis $|q-1|<\omega$, so we will assume this hypothesis starting from Hypothesis 5.3.6.

— Step 1 : By [DV04, 3.6], the equation $\sigma_{q}-a^{-}(T)$ (resp. $\sigma_{q}-a^{+}(T)$ ) has a convergent solution at $\infty$ (resp. at 0$)$, hence $\operatorname{Ray}\left(\sigma_{q}-a^{-}(T), \rho\right)=\rho$, for large values of $\rho\left(\operatorname{resp}\right.$. $\operatorname{Ray}\left(\sigma_{q}-a^{-}(T), \rho\right)=\rho$ for $\rho$ close to 0$)$. Let $R^{0}$ be as in Lemma 5.1.5,

$$
\begin{aligned}
& R^{-}:=\operatorname{Ray}\left(\sigma_{q}-a^{-}(T), 1\right), \\
& R^{+}:=\operatorname{Ray}\left(\sigma_{q}-a^{+}(T), 1\right) .
\end{aligned}
$$

- Step 2: We have $R^{+}=R^{-}$and $R^{0} \geqslant R^{-}=R^{+}$(as in the following picture in which $\left.R:=R^{-}=R^{+}\right)$.

We set $r:=\log (\rho)$, and $R(r):=\log \left(\operatorname{Ray}\left(\sigma_{q}-a(T), \rho\right) / \rho\right)$.

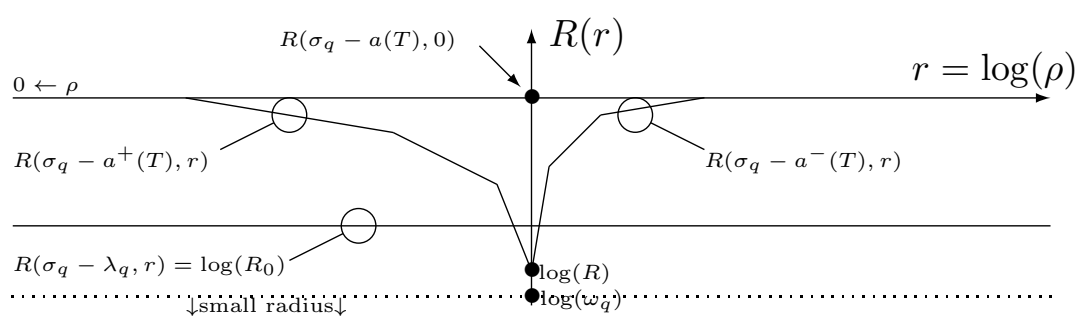

- Step 3: We have $R \geqslant \omega_{q}$.

Indeed, if $R^{-}=R^{+}<\omega_{q}$, then, by the small radius Lemma 5.1.3, $\left|a^{-}(T)-1\right|_{1}>|q-1|$ and $\left|a^{+}(T)-1\right|_{1}>|q-1|$. We shall now show that this implies that $|a(T)-1|_{1}>|q-1|$, which is in contradiction with the small radius lemma, since the equation $\sigma_{q}-a(T)$ is solvable.

Lemma 5.3.3. Let $(R,||$.$) be an ultrametric valued ring. Let h^{-}, h^{+} \in R$ be two elements satisfying $\left|h^{-}\right|<1$, and $\left|h^{-}+h^{+}\right|=\sup \left(\left|h^{-}\right|,\left|h^{+}\right|\right)$. Then

$$
\left|h^{-}+h^{+}+h^{-} h^{+}\right|=\sup \left(\left|h^{-}\right|,\left|h^{+}\right|\right) \text {. }
$$


Proof. If $\left|h^{+}\right|>\left|h^{-}\right|$, then $\left|h^{-}+h^{+}+h^{-} h^{+}\right|=\left|h^{+}\right|$. If $\left|h^{+}\right| \leqslant\left|h^{-}\right|<1$, then $\left|h^{-} h^{+}\right|<\left|h^{-}\right|=$ $\max \left(\left|h^{-}\right|,\left|h^{+}\right|\right)=\left|h^{-}+h^{+}\right|$.

Proof of Step 3: Write $a^{-}(T)=1+h_{q}^{-}(T)$ and $\lambda_{q} \cdot a^{+}(T)=1+\left(\lambda_{q}-1\right)+\lambda_{q} \cdot h_{q}^{+}(T)$. Namely, in the notations of Theorem 4.0.14, we have $h_{q}^{-}(T)=\sum_{i \leqslant-1} \alpha_{i} T^{i}$ and $h_{q}^{+}(T)=\sum_{i \geqslant 1} \alpha_{i} T^{i}$. We apply Lemma 5.3 .3 to the field $R:=\mathcal{E}_{K}, h^{-}:=h_{q}^{-}(T)$ and $h^{+}:=\left(\lambda_{q}-1\right)+\lambda_{q} h_{q}^{+}(T)$. Indeed $\left|h^{-}+h^{+}\right|_{1}=\sup \left(\left|h^{-}\right|_{1},\left|h^{+}\right|_{1}\right)$, and $\left|h^{-}\right|_{1}<1$ by Lemma 4.0.15. Lemma 5.3.3 then implies

$$
|a(T)-1|_{1}=\left|\left(1+h^{-}\right)\left(1+h^{+}\right)-1\right|_{1}=\left|h^{-}+h^{+}+h^{-} h^{+}\right|_{1}=\sup \left(\left|h^{-}\right|_{1},\left|h^{+}\right|_{1}\right) .
$$

Now, if $R^{-}<\omega_{q}$, then $\left|a^{-}(T)-1\right|>|q-1|$, that is $\left|h^{-}(T)\right|>|q-1|$. Hence $|a(T)-1|_{1}>|q-1|$, which implies that the radius of $\sigma_{q}-a(T)$ is small (cf. Lemma 5.1.3). Since, by assumption, $\operatorname{Ray}\left(\sigma_{q}-a(T), 1\right)=1$, this is absurd and then $R \geqslant \omega_{q}$.

- Step 4: We have $R>\omega_{q}$.

Since $R=R^{-}$it is enough to show that $R^{-}>\omega_{q}$. By Lemma 5.3.4 below we have $\left|a^{-}-1\right|<|q-1|$. On the other hand Lemma 5.3.5 proves that this implies $R^{-}>\omega_{q}$.

Lemma 5.3.4 (q-analogue of Lemma 2.1.3). Assume that the Motzkin decomposition of a $(T) \in \mathcal{E}_{K}$ is $a(T):=\lambda_{q} a^{-}(T) a^{+}(T)$, with $\left|\lambda_{q}-1\right|<1$. If $\operatorname{Ray}\left(\sigma_{q}-a(T), 1\right)>\omega_{q}$, then we have $a^{-}(T)=$ $1+h_{q}^{-}(T)$, where $h_{q}^{-}(T)=\sum_{i \leqslant-1} \alpha_{i} T^{i}$ satisfies $\left|h_{q}^{-}\right|_{1}<|q-1|$.

Proof. Consider the operator $d_{q}-g_{[1]}(T)$, with $g_{[1]}(T):=\frac{a(T)-1}{(q-1) T}$, and write

$$
g_{[1]}(T)=\frac{a^{-}(T)-1}{(q-1) T}+a^{-}(T) \frac{\lambda_{q} a^{+}(T)-1}{(q-1) T}=g_{[1]}^{-}(T)+a^{-}(T) \frac{\lambda_{q} a^{+}(T)-1}{(q-1) T},
$$

with $g_{[1]}^{-}(T):=\frac{a^{-}(T)-1}{(q-1) T}$. Since $\operatorname{Ray}\left(d_{q}-g_{[1]}(T), 1\right)>\omega_{q}$, hence, by (5.8) and Lemma 5.1.1, one has $\lim _{s \rightarrow \infty}\left|g_{[s]}(T)\right|_{1}=0$. In particular $\left|g_{[s]}(T)\right|_{1}<1$, for some $s \geqslant 1$. Moreover, by the Small Radius Lemma 5.1.3, we have $\left|g_{[1]}(T)\right|_{1} \leqslant 1$. These facts imply our claim in the following way.

By contrapositive, suppose that $\left|a^{-}(T)-1\right|_{1} \geqslant|q-1|$. Our assumption $\operatorname{Ray}\left(\sigma_{q}-a(T), 1\right)>\omega_{q}$ is enough to obtain Steps 1,2,3. In particular Step 3 says $\operatorname{Ray}\left(\sigma_{q}-a^{-}(T), 1\right) \geqslant \omega_{q}$. Then, by Lemma 5.1.3, $\left|g_{[1]}^{-}(T)\right|_{1} \leqslant 1$, and hence $\left|a^{-}(T)-1\right|_{1}=|q-1|$. This means $\left|g_{[1]}^{-}(T)\right|_{1}=1$.

We now look to $g_{[1]}$ and get a contradiction exploiting (5.18) and the fact that $\left|g_{[1]}^{-}(T)\right|_{1}=1$. Namely, write as usual $a^{-}(T)=1+\sum_{i \geqslant 1} \alpha_{-i} T^{-i}$. Let $-d \leqslant-1$ be the smallest index such that $\left|\alpha_{-d}\right|=|q-1|$. Observing equation (5.18) we see that by Lemma 4.0.15 the reduction $a^{-}(T)$ is 1 , and the reduction of $\frac{\lambda_{q} a^{+}(T)-1}{(q-1) T}$ lies in $t^{-1} k[[t]]$, so the reduction of $g_{[1]}(T)$ in $k((t))$ is of the form $\overline{g_{[1]}(T)}=\bar{\alpha} t^{-d-1}+\left(\right.$ terms of higher degree), where $\bar{\alpha}$ is the reduction of $\alpha_{-d} /(q-1)$.

A simple induction on the equation $g_{[s+1]}=d_{q}\left(g_{[s]}\right)+\sigma_{q}\left(g_{[s]}\right) g_{[1]}$ shows that $\overline{g_{[s]}(T)}=\bar{\alpha}^{s} t^{(-d-1) s}+$ (terms of higher degree), this is in contradiction with the fact that $\left|g_{[s]}(T)\right|_{1}<1$, for some $s \geqslant 1$.

Lemma 5.3.5 (q-analogue of Lemma 2.1.2). Let $q \in \mathrm{D}^{-}(1,1)$. Let $\Delta_{q}-g(T), g(T) \in \mathcal{E}_{K}$, be some equation. Suppose that $|g(T)|_{1} \leqslant 1$. Then Ray $\left(\Delta_{q}-g(T), 1\right)>\omega_{q}$ if and only if $\left|g_{[s]}(T)\right|<1$, for some $s \geqslant 1$.

Proof. Condition $|g(T)|_{1} \leqslant 1$ guarantee that $n \mapsto\left|g_{[n]}\right|_{1}$ is decreasing. Indeed, $\left|g_{[1]}\right|_{1}=\left|T^{-1} g(T)\right|_{1} \leqslant$ 1 and inductively $\left|g_{[n+1]}\right|_{1}=\left|d_{q}\left(g_{[n]}\right)+\sigma_{q}\left(g_{[n]}\right) g_{[1]}\right|_{1} \leqslant \sup \left(\left|g_{[n]}\right|{ }_{1},\left|g_{[n]} g_{[1]}\right|_{1}\right)=\left|g_{[n]}\right|_{1} \sup \left(1,\left|g_{[1]}\right|_{1}\right)=$ $\left|g_{[n]}\right|_{1}$. So if $\operatorname{Ray}\left(d_{q}-g(T), 1\right)>\omega_{q}$, it follows from (5.8) that $\lim _{n}\left|g_{[n]}(T)\right|_{1}=0$.

Assume now that $\left|g_{[n]}\right|_{1}<1$, for some $n \geqslant 1$. Since the sequence $n \mapsto\left|g_{[n]}\right|_{1}$ is decreasing, there exists $h>0$ such that $\left|g_{\left[p^{h}\right]}\right|_{1}<1$. We now fix such an $h$, and we obtain an estimation of 


\section{AndRea Pulita}

$\operatorname{Ray}\left(d_{q}-g(T), 1\right)$. By [DV04, 1.2.2], one has

$$
d_{q}^{(m+1) p^{h}}(y)=d_{q}^{p^{h}}\left(d_{q}^{m p^{h}}(y)\right)=\sum_{r=0}^{p^{h}}\left(\begin{array}{c}
p^{h} \\
r
\end{array}\right)_{q} d_{q}^{r}\left(g_{\left[m p^{h}\right]}\right) \cdot \sigma_{q}^{r}\left(g_{\left[p^{h}-r\right]}\right) \sigma_{q}^{r}(y) .
$$

Then $g_{\left[(m+1) p^{h}\right]}=\sum_{r=0}^{p^{h}}\left(\begin{array}{c}p^{h} \\ r\end{array}\right)_{q} d_{q}^{r}\left(g_{\left[m p^{h}\right]}\right) \cdot \sigma_{q}^{r}\left(g_{\left[p^{h}-r\right]}\right) a(T) a(q T) \cdots a\left(q^{r-1} T\right)$. Now for all $j \geqslant 0$ one has $\left|a\left(q^{j} T\right)\right|_{1}=|a(T)|_{1}=1$, and on the other hand $\left|d_{q}^{k}(f)\right|_{1} \leqslant\left|[k]_{q}^{!}\right||f|_{1}$ (cf. Lemma 5.1.2). Moreover $\left|\left(\begin{array}{c}p^{h} \\ r\end{array}\right)_{q}\right|=\left|\left[p^{h}\right]_{q}\left[p^{h}-1\right]_{q} \cdots\left[p^{h}-r+1\right]_{q}\right| /\left|[r]_{q}^{!}\right|$, where $[n]_{q}:=\frac{q^{n}-1}{q-1}$. Since $\left|\left[p^{h}\right]_{q}\right|<\left|[p]_{q}\right|$, we obtain

$$
\left|g_{\left[(m+1) p^{h}\right]}\right|_{1} \leqslant \sup \left(\left|[p]_{q}\right|,\left|g_{\left[p^{h}\right]}\right|_{1}\right) \cdot\left|g_{\left[m p^{h}\right]}\right|_{1} .
$$

We deduce that for all $m \geqslant 1$ one has $\left|g_{\left[m p^{h}\right]}\right|_{1} \leqslant s^{m}$, where $s:=\sup \left(\left|[p]_{q}\right|,\left|g_{\left[p^{h}\right]}\right|_{1}\right)<1$.

Now we obtain a similar estimation for all $n \geqslant p^{h}$. We let $m(n):=\left[n / p^{h}\right] \geqslant 1$, where $[a]$ is the greatest integer smaller than or equal to $a$. Then $m(n) p^{h} \leqslant n$ and $\left|g_{[n]}\right|_{1} \leqslant\left|g_{\left[m(n) p^{h}\right]}\right|_{1} \leqslant s^{m(n)}$.

Finally we now obtain the required estimation. We have

$$
\left|\frac{g_{[n]}}{[n]_{q}^{!}}\right|_{1}^{\frac{1}{n}} \leqslant \frac{s^{m(n) / n}}{\left|[n]_{q}^{!}\right|^{1 / n}} \leqslant \frac{s^{1 / p^{h}}}{\left|[n]_{q}^{!}\right|^{1 / n}} \stackrel{n \mapsto \infty}{\longrightarrow} \frac{s^{1 / p^{h}}}{\omega_{q}} .
$$

By (5.8), this gives $\operatorname{Ray}\left(\Delta_{q}-g_{[1]}, 1\right) \geqslant \omega_{q} / s^{1 / p^{h}}>\omega_{q}$.

Hypothesis 5.3.6. From now on we will suppose that $|q-1|<\omega$. This implies $\omega_{q}=\omega$.

Hypothesis 5.3.6 is necessary to have Theorem [DV04]: the antecedent by Frobenius.

- Step 5: Since $|q-1|<1$, and since $R>\omega$, then, by [DV04], we can take the antecedent by Frobenius of $\sigma_{q}-a^{-}(T), \sigma_{q}-a^{+}(T)$ and $\sigma_{q}-\lambda_{q}$.

More precisely, there exist a finite extension $K^{(1)} / K$, an $f^{+}(T)=\sum_{i \geqslant 0} b_{i}^{+} T^{i} \in \mathcal{A}_{K^{(1)}}\left(\left[0,1[)^{\times}\right.\right.$, $f^{-}(T)=\sum_{i \leqslant 0} b_{i}^{-} T^{i} \in \mathcal{A}_{K^{(1)}}([1, \infty])^{\times}$, and there are functions $a^{(1),-}(T)=\sum_{i \leqslant 0} \alpha_{i}^{(1),-} T^{i} \in \mathcal{E}_{K^{(1)}}$, $a^{(1),+}(T)=\sum_{i \geqslant 0} \alpha_{i}^{(1),+} T^{i} \in \mathcal{E}_{K^{(1)}}$, and $\lambda_{q}^{(1)} \in K^{(1)}$ such that

$$
\begin{aligned}
\left(\lambda_{q}^{(1)}\right)^{p} & =\lambda_{q} ; \\
a^{(1),-}\left(T^{p}\right)^{\sigma} \cdot a^{(1),-}\left(q \cdot T^{p}\right)^{\sigma} \cdots a^{(1),-}\left(q^{p-1} T^{p}\right)^{\sigma} & =a^{-}(T) \cdot \frac{f^{-}(q \cdot T)}{f^{-}(T)} ; \\
a^{(1),+}\left(T^{p}\right)^{\sigma} \cdot a^{(1),+}\left(q \cdot T^{p}\right)^{\sigma} \cdots a^{(1),+}\left(q^{p-1} T^{p}\right)^{\sigma} & =a^{+}(T) \cdot \frac{f^{+}(q \cdot T)}{f^{+}(T)},
\end{aligned}
$$

where, for all functions $a(T):=\sum \alpha_{i} T^{i} \in \mathcal{E}_{K}$, we let $a(T)^{\sigma}:=\sum \sigma\left(\alpha_{i}\right) T^{i}$.

These conditions imply immediately that $b_{0}^{+} \neq 0, b_{0}^{-} \neq 0$, and that $\left.f^{+}(q T) / f^{+}(T)\right)=1+u_{1} T+$ $u_{2} T^{2}+\cdots$, and $f^{-}(q T) / f^{-}(T)=1+u_{-1} T^{-1}+u_{-2} T^{-2}+\cdots$. Since $a^{-}(T)=1+\alpha_{-1} T^{-1}+\cdots$, and $a^{+}(T)=1+\alpha_{1} T+\cdots$, this implies that $\alpha_{0}^{(1),+}=1$ and $\alpha_{0}^{(1),-}=1$. Hence the function

$$
a^{(1)}(T):=\lambda_{q}^{(1)} \cdot a^{(1),-}(T) \cdot a^{(1),+}(T)
$$

lies in $\mathcal{E}_{K}$, and it is the Motzkin decomposition of Theorem 4.0.17. Observe now that both $f^{-}$and $f^{+}$belong to $\mathcal{E}_{K}^{\times}$, hence $\sigma_{q}-a^{(1)}(T)$ is an antecedent of Frobenius of $\sigma_{q}-a(T)$ over $\mathcal{E}_{K}$, and it is then solvable. 
— Step 6: Steps 1, 2, 3, 4 are still true for the antecedent. In particular if

$$
\begin{aligned}
R^{-}(1) & :=\operatorname{Ray}\left(\partial_{T}-g^{(1),-}(T), 1\right), \\
R^{+}(1) & :=\operatorname{Ray}\left(\partial_{T}-g^{(1),+}(T), 1\right), \\
R^{0}(1) & :=\operatorname{Ray}\left(\partial_{T}-b_{0}, 1\right) .
\end{aligned}
$$

we must have $R^{-}(1)=R^{+}(1)>\omega$. Let $R(1):=R^{-}(1)=R^{+}(1)$, then $R(1)=R^{1 / p}$, by the property of the antecedent. This implies $R>\omega^{1 / p}$.

Now the process can be iterated since $R(1)>\omega$, and we can again consider the antecedent. This shows that $R>\omega^{1 / p^{h}}$, for all $h \geqslant 0$, that is $R=1$. Proposition 5.3.2 hence follows.

Corollary 5.3.7 (q-analogue of 2.1.4). Let $q \in \mathrm{D}^{-}(1,1)$. Let $\sigma_{q}-a(T)$ be a solvable differential equation. Let $a(T)=\lambda \cdot a^{-}(T) \cdot a^{+}(T)$ be the Motzkin decomposition of $a(T)$. Then $\lambda=q^{a_{0}}$, for some $a_{0} \in K$. Moreover, this operator is isomorphic to $\sigma_{q}-\lambda \cdot a^{-}(T)$.

Proof. See the proof of 2.1.4.

Remark 5.3.8. The unique obstruction to generalize Proposition 5.3.2 and Corollary 5.3.7 to the case $|q-1|<1$ is represented by the so called Weak Frobenius structure for q-difference modules over a disk with $|q-1|<1$. This is proved in [DV04] in the case $|q-1|<\omega$.

The assumption $|q-1|<\omega$ is also used in the sequel, where we consider logarithms of the exponentials. E.g. see Step 0 of Lemma 5.4.1.

\subsection{Criterion of Solvability}

Lemma 5.4.1 (q-analogue of 2.2.1). Let $|q-1|<\omega$. Suppose that $a(T)=a^{+}(T)$ is the Motzkin decomposition of $a(T)$. Write $a^{+}(T)=\exp \left(\sum_{i \geqslant 1} a_{i} T^{i}\right) \in \mathcal{A}\left(\left[0,1[)^{\times}\right.\right.$(cf. the settings of 5.2). Then the $q$-difference equation $\sigma_{q}-a^{+}(T)$ is solvable if and only if there exists a family $\left\{\boldsymbol{\lambda}_{n}\right\}_{n \in \mathbb{J}}$, where $\boldsymbol{\lambda}_{n} \in \mathbf{W}\left(\mathscr{O}_{K}\right)$ has phantom components $\phi_{n}=\left(\phi_{n, 0}, \phi_{n, 1}, \ldots\right)$ satisfying

$$
a_{n p^{m}}=\frac{\left(q^{n p^{m}}-1\right)}{p^{m}} \cdot \phi_{n, m}, \quad \text { for all } n \in \mathbb{J}, m \geqslant 0
$$

for all $n \in \mathbb{J}$, and all $m \geqslant 0$. In other words, the formal solution of the equation $\sigma_{q}(y)=$ ay is (5.12)

$$
y(T)=E\left(\sum_{n \in \mathbb{J}} \boldsymbol{\lambda}_{n} T^{n}, 1\right):=\exp \left(\sum_{n \in \mathbb{J}} \sum_{m \geqslant 0} \phi_{n, m} \frac{T^{n p^{m}}}{p^{m}}\right) .
$$

Proof. The formal series $E\left(\sum_{n \in \mathbb{J}} \boldsymbol{\lambda}_{n} T^{n}, 1\right)$ belongs to $1+T \cdot \mathfrak{p}_{K}[[T]] \subset \mathcal{E}_{K}$, and it is solution of the equation $L:=\sigma_{q}-\exp \left(\sum_{n \in \mathbb{J}} \sum_{m \geqslant 0} \phi_{n, m}\left(q^{n p^{m}}-1\right) T^{n p^{m}}\right)$. Since this exponential converges in the open unit disk, then $\operatorname{Ray}(L, \rho)=\rho$, for all $\rho<1$. Hence, by continuity of the radius, $\operatorname{Ray}(L, 1)=1$ and $L$ is solvable.

Conversely, suppose that $\sigma_{q}-a^{+}(T)$ is solvable, then the Witt vectors $\boldsymbol{\lambda}_{n}=\left(\lambda_{n, 0}, \lambda_{n, 1}, \ldots\right)$ are defined by the relation (5.25). For example, for all $n \in \mathbb{J}$ we have

$$
\lambda_{n, 0}=\frac{a_{n}}{\left(q^{n}-1\right)} \quad, \quad \lambda_{n, 1}=\frac{1}{p}\left(\frac{p \cdot a_{n p}}{\left(q^{n p}-1\right)}-\left(\frac{a_{n}}{\left(q^{n}-1\right)}\right)^{p}\right) .
$$

We must show that $\left|\lambda_{n, m}\right| \leqslant 1$, for all $n \in \mathbb{J}, m \geqslant 0$.

— Step $0:$ We have $\left|\lambda_{n, 0}\right|=\left|\phi_{n, 0}\right| \leqslant 1$ for all $n \in \mathbb{J}$.

This results by the small radius Lemma 5.1.3 as follows: denote the argument of the ex- 


\section{Andrea Pulita}

ponential $a^{+}(T)$ by $\phi_{q}^{+}(T):=\sum_{n \in \mathbb{J}} \sum_{m \geqslant 0} \phi_{n, m}\left(q^{n p^{m}}-1\right) T^{p^{m}} / p^{m}$. By Lemma 5.1.3, one has $\left|a^{+}(T)-1\right|_{1}=\left|\exp \left(\phi_{q}^{+}\right)-1\right|_{1} \leqslant|q-1|$. Since $|q-1|<\omega$, then $\left|\exp \left(\phi_{q}^{+}\right)-1\right|_{1}<\omega$, hence $\phi_{q}^{+}=\log \left(\exp \left(\phi_{q}^{+}\right)\right)$and $\left|\phi_{q}^{+}\right|_{1}=\left|\exp \left(\phi_{q}^{+}\right)-1\right|_{1} \leqslant|q-1|$. This implies $\left|\phi_{n, m}\left(q^{n p^{m}}-1\right) / p^{m}\right| \leqslant|q-1|$, for all $n \in \mathbb{J}$ and all $m \geqslant 0$. In particular, for $m=0$ we have $\left|\lambda_{n, 0}\right|=\left|\phi_{n, 0}\right| \leqslant 1$, for all $n \in \mathbb{J}$.

— Step 1 : By Step 0 the exponential

$$
E\left(\sum_{n \in \mathbb{J}}\left(\lambda_{n, 0}, 0,0, \ldots\right) T^{n}, 1\right)=\exp \left(\sum_{n \in \mathbb{J}} \sum_{m \geqslant 0} \lambda_{n, 0}^{p^{m}} \frac{T^{n p^{m}}}{p^{m}}\right)
$$

converges in the unit disk and is solution of the operator $Q^{(0)}:=\sigma_{q}-a^{(0)}(T)$, with $a^{(0)}(T)=$ $\exp \left(\sum_{n \in \mathbb{J}} \sum_{m \geqslant 0} \lambda_{n, 0}^{p^{m}}\left(q^{n p^{m}}-1\right) \frac{T^{n p^{m}}}{p^{m}}\right) . Q^{(0)}$ is then solvable.

- Step 2 : The tensor product operator $\sigma_{q}-\left(a^{+}(T) / a^{(0)}(T)\right)$ is again solvable. We have explicitly

$$
\frac{a^{+}(T)}{a^{(0)}(T)}=\exp \left(\sum_{n \in \mathbb{J}} \sum_{m \geqslant 0}\left(\phi_{n, m}-\lambda_{n, 0}^{p^{m}}\right)\left(q^{n p^{m}}-1\right) \frac{T^{n p^{m}}}{p^{m}}\right) .
$$

This operator corresponds to the family of Witt vectors $\left\{\boldsymbol{\lambda}_{n}-\left(\lambda_{n, 0}, 0,0, \ldots\right)=\left(0, \lambda_{n, 1}, \lambda_{n, 2}, \ldots\right)\right\}_{n \in \mathbb{J}}$. Observe that the coefficient corresponding to $m=0$ is equal to 0 , for all $n \in \mathbb{J}$. This leads us to compute easily the "antecedent by ramification" of $\sigma_{q}-a^{+}(T) / a^{(0)}(T)$, namely this antecedent is given by $\sigma_{q}-a^{(1)}(T)$, with

$$
a^{(1)}(T):=\exp \left(\sum_{n \in \mathbb{J}} \sum_{m \geqslant 0}\left(\phi_{n, m}-\lambda_{n, 0}^{p^{m}}\right)\left(q^{n p^{m}}-1\right) \frac{(q-1)}{\left(q^{p}-1\right)} \frac{T^{n p^{m-1}}}{p^{m}}\right) .
$$

In other words, we have

$$
a^{(1)}\left(T^{p}\right) \cdot a^{(1)}\left(q T^{p}\right) \cdot a^{(1)}\left(q^{2} T^{p}\right) \cdots a^{(1)}\left(q^{p-1} T^{p}\right)=\frac{a^{+}(T)}{a^{(0)}(T)} .
$$

- Step 3 : The antecedent is again solvable, hence, as in Step 0, we find $\left|\phi_{n, 1}-\lambda_{n, 0}^{p}\right| \leqslant\left|q^{p}-1\right|=$ $|p|$, which implies $\left|\lambda_{n, 1}\right| \leqslant 1$.

The process can be iterated indefinitely.

Remark 5.4.2 (q-analogue of Remark 2.2.2). We shall now consider the general case of an equation $\sigma_{q}-a(T)$, with $a(T)=\lambda \cdot a^{-}(T) a^{+}(T) \in \mathcal{E}_{K}$, and get a criteria of solvability. We proceed as in Remark 2.2.2. Suppose given two families $\left\{\boldsymbol{\lambda}_{-n}\right\}_{n \in \mathbb{J}}$ and $\left\{\boldsymbol{\lambda}_{n}\right\}_{n \in \mathbb{J}}$, with $\boldsymbol{\lambda}_{n} \in \mathbf{W}\left(\mathscr{O}_{K}\right)$. By Lemma 5.4.4 below, $a^{+}(T)$ belongs always to $\mathcal{E}_{K}$. On the other hand, we will prove (cf. Lemma 5.4.5) that the series $a^{-}(T)$ belongs to $\mathcal{E}_{K}$ if and only if the family $\left\{\boldsymbol{\lambda}_{-n}\right\}_{n \in \mathbb{J}}$ belongs to $\operatorname{Conv}\left(\mathcal{E}_{K}\right)$ (cf. Definition 2.2.5).

Notation 5.4.3. Let $\sigma_{q}-a(q, T), a(q, T) \in \mathcal{E}_{K}$ be a solvable differential equation. Let $a(q, T):=$ $q^{a_{0}} \cdot a^{-}(q, T) \cdot a^{+}(q, T), a_{0} \in \mathbb{Z}_{p}$, be the Motzkin decomposition of a $(q, T)$. In the notations of Lemma 5.4 .1 we can write

$$
\begin{aligned}
a^{-}(q, T)= & \exp \left(\phi_{q}^{-}(T)\right) \quad, \quad a^{+}(q, T)=\exp \left(\phi_{q}^{+}(T)\right), \\
\phi_{q}^{-}(T) & :=\sum_{n \in \mathbb{J}} \sum_{m \geqslant 0} \phi_{-n, m}\left(q^{-n p^{m}}-1\right) \frac{T^{-n p^{m}}}{p^{m}}, \\
\phi_{q}^{+}(T) & :=\sum_{n \in \mathbb{J}} \sum_{m \geqslant 0} \phi_{n, m}\left(q^{n p^{m}}-1\right) \frac{T^{n p^{m}}}{p^{m}} .
\end{aligned}
$$


Solvability of RANK one $p$-ADIC Differential AND $q$-DIfFEREnCE EQUATIONS Over the Amice RING

For all $n \in \mathbb{J}$ we denote by $\boldsymbol{\lambda}_{n}, \boldsymbol{\lambda}_{-n} \in \mathbf{W}(K)$ the Witt vectors with phantom vectors $\left\langle\phi_{n, 0}, \phi_{n, 1}, \ldots\right\rangle$ and $\left\langle\phi_{-n, 0}, \phi_{-n, 1}, \ldots\right\rangle$ respectively. In other words, the solution of $\sigma_{q}-a(q, T)$ can be represented by the symbol

$$
y(T):=T^{a_{0}} \cdot \exp \left(\sum_{n \in \mathbb{J}} \sum_{m \geqslant 0} \phi_{-n, m} \frac{T^{-n p^{m}}}{p^{m}}\right) \cdot \exp \left(\sum_{n \in \mathbb{J}} \sum_{m \geqslant 0} \phi_{n, m} \frac{T^{n p^{m}}}{p^{m}}\right),
$$

as well as for differential equations.

Lemma 5.4.4. Let $|q-1|<\omega$. Let $\left\{\boldsymbol{\lambda}_{n}\right\}_{n \in \mathbb{J}}$ be a family of Witt vectors such that $\boldsymbol{\lambda}_{n} \in \mathbf{W}\left(\mathscr{O}_{K}\right)$. Then $a^{+}(T)$ belongs to $\mathcal{E}_{K}$.

Proof. We use the notations of [Pul07]. Let $P(X)=(X+1)^{p}-1$ be the Lubin-Tate series corresponding to the formal multiplicative group $\hat{\mathbb{G}}_{m}$. The phantom vector of $\left[q^{n}-1\right]_{P} \in \mathbf{W}\left(\mathscr{O}_{K}\right)$ is then $\left\langle q^{n}-1, q^{n p}-1, q^{n p^{2}}-1, \cdots\right\rangle$, for all $n \in \mathbb{Z}$. Then, for all $n \in \mathbb{J}$, the phantom vector of $\left[q^{n}-1\right]_{P} \cdot \boldsymbol{\lambda}_{n}$ is

$$
\left\langle\left(q^{n}-1\right) \phi_{n, 0},\left(q^{n p}-1\right) \phi_{n, 1},\left(q^{n p^{2}}-1\right) \phi_{n, 2}, \ldots\right\rangle .
$$

Hence we can express $a^{+}(q, T)$ as a product of Artin-Hasse exponentials

$$
a^{+}(q, T)=\prod_{n \in \mathbb{J}} E\left(\left[q^{n}-1\right]_{P} \cdot \boldsymbol{\lambda}_{n}, T\right) .
$$

Since $\left[q^{n}-1\right]_{P} \cdot \boldsymbol{\lambda}_{n} \in \mathbf{W}\left(\mathscr{O}_{K}\right)$, then for all $n \in \mathbb{J}$ the Artin-Hasse exponential $E\left(\left[q^{n}-1\right]_{P} \cdot \boldsymbol{\lambda}_{n}, T\right)$ belongs to $1+T \mathscr{O}_{K}[[T]]$, which is contained in $\mathcal{E}_{K}$.

Lemma 5.4.5 (q-analogue of Proposition 2.2.3). Let $|q-1|<\omega$. Let $\left\{\boldsymbol{\lambda}_{-n}\right\}_{n \in \mathbb{J}} \in \mathbf{W}\left(\mathscr{O}_{K}\right)$ be a family of Witt vectors. Then the following assertions are equivalent:

(1) The series $a^{-}(T)=\exp \left(\phi_{q}^{-}(T)\right)$ belongs to $\mathcal{E}_{K}$;

(2) $\phi_{q}^{-}(T)$ belongs to $\mathcal{E}_{K}$

(3) $\left\{\boldsymbol{\lambda}_{-n}\right\}_{n \in \mathbb{J}} \in \operatorname{Conv}(\mathcal{E})$ (cf. Definition 2.2.5).

Proof. The equivalence $(2) \Leftrightarrow(3)$ follows from Proposition 2.2.3.

We firstly observe that since by assumption we have $\boldsymbol{\lambda}_{-n} \in \mathbf{W}\left(\mathscr{O}_{K}\right)$, then $\left|\phi_{-n, m}\right| \leqslant 1$ and so

$$
\left|\left(q^{-n p^{m}}-1\right) \phi_{-n, m} p^{-m}\right|=|q-1| \cdot\left|\phi_{-n, m}\right| \leqslant|q-1|<\omega .
$$

Hence $\left|\phi_{q}^{-}(T)\right|_{1} \leqslant|q-1|<\omega$.

Now assume that $\phi_{q}^{-}(T) \in \mathcal{E}_{K}$. Since the exponential series converges in the disk $D_{\mathcal{E}_{K}}^{-}(0, \omega):=$ $\left\{\left.f \in \mathcal{E}_{K}|| f\right|_{1}<\omega\right\}$, then $\exp \left(\phi_{q}^{-}(T)\right) \in \mathcal{E}_{K}$.

Conversely, assume that $\exp \left(\phi_{q}^{-}(T)\right) \in \mathcal{E}_{K}$. Since, for all $\rho>1,\left|\phi_{q}^{-}(T)\right|_{\rho}<|q-1|$, then $\phi_{q}^{-}(T) \in \mathrm{D}_{\mathcal{A}_{K}([\rho, \infty])}^{-}(0, \omega):=\left\{\left.f \in \mathcal{A}_{K}([\rho, \infty])|| f\right|_{\rho}<\omega\right\}$, and hence $\exp \left(\phi_{q}^{-}(T)\right)$ converge in $\mathcal{A}_{K}([\rho, \infty])$, for all $\rho>1$. Moreover, $\left|\exp \left(\phi_{q}^{-}(T)\right)-1\right|_{\rho}=\left|\phi_{q}^{-}(T)\right|_{\rho} \leqslant|q-1|<\omega$, for all $\rho>1$. By continuity, we have $\left|\exp \left(\phi_{q}^{-}(T)\right)\right|_{1}=\left|\phi_{q}^{-}(T)\right|_{1} \leqslant|q-1|<\omega$. Now the logarithm converges in the disk $D_{\mathcal{E}_{K}}\left(1,1^{-}\right):=\left\{\left.f \in \mathcal{E}_{K}|| f\right|_{1}<1\right\}$, hence $\phi_{q}^{-}(T)=\log \exp \left(\phi_{q}^{-}(T)\right)$. Then $\phi_{q}^{-}(T)$ belongs to $\mathcal{E}_{K}$.

Corollary 5.4.6 (Criterion of solvability for $q$-difference equations). The equation $\sigma_{q}-a(q, T)$, with $a(q, T)=\lambda_{q} T^{N} a^{-}(q, T) a^{+}(q, T)$, with

$$
a^{-}(T):=\exp \left(\sum_{i \leqslant-1} a_{i} T^{i}\right) \quad, \quad a^{+}(T):=\exp \left(\sum_{i \geqslant 1} a_{i} T^{i}\right),
$$

is solvable if and only if the following conditions are verified 


\section{Andrea Pulita}

i) $\lambda=q^{a_{0}}$, with $a_{0} \in \mathbb{Z}_{p}$;

ii) $N=0$;

iii) There exist two families $\left\{\boldsymbol{\lambda}_{-n}\right\}_{n \in \mathbb{J}}$ and $\left\{\boldsymbol{\lambda}_{n}\right\}_{n \in \mathbb{J}}$, with $\boldsymbol{\lambda}_{-n}, \boldsymbol{\lambda}_{n} \in \mathbf{W}\left(\mathscr{O}_{K}\right)$, for all $n \in \mathbb{J}$, such that

$$
a_{-n p^{m}}=\frac{\left(q^{-n p^{m}}-1\right)}{p^{m}} \cdot \phi_{-n, m} \quad, \quad a_{n p^{m}}=\frac{\left(q^{n p^{m}}-1\right)}{p^{m}} \cdot \phi_{n, m},
$$

for all $n \in \mathbb{J}$ and all $m \geqslant 0$;

iv) $\left\{\boldsymbol{\lambda}_{-n}\right\}_{n \in \mathbb{J}} \in \operatorname{Conv}(\mathcal{E})$.

In other words, the formal solution of this equation can be represented by the symbol (5.31) in which the family $\left\{\boldsymbol{\lambda}_{-n}\right\}_{n \in \mathbb{J}}$ belongs to $\operatorname{Conv}\left(\mathcal{E}_{K}\right)$, and $a(T)=\exp \left(\phi_{q}^{-}(T)\right) \cdot q^{a_{0}} \cdot \exp \left(\phi_{q}^{+}(T)\right)$, where $\phi_{q}^{-}(T)$, $\phi_{q}^{+}(T)$ are defined in (5.29) and (5.30).

Corollary 5.4.7 (canonical extension for $q$-difference). Let $\sigma_{q}-\operatorname{Mod}\left(\mathcal{A}_{K}([1, \infty])\right)_{r k=1}^{\text {sol }}$ be the category of rank one $\sigma_{q}$-modules over $\mathcal{A}_{K}([1, \infty])$, solvable at all $\rho \geqslant 1$. The scalar extension functor $\sigma_{q}-\operatorname{Mod}\left(\mathcal{A}_{K}([1, \infty])\right)_{r k=1}^{\text {sol }} \rightarrow \sigma_{q}-\operatorname{Mod}\left(\mathcal{E}_{K}\right)_{r k=1}^{\text {sol }}$ is an equivalence.

Proof. The proof is analogous to the proof of Corollary 2.2.7.

Remark 5.4.8 (Strong confluence). The q-deformation and q-confluence equivalences of [Pul08] do not hold over the ring $\mathcal{E}_{K}$. Indeed those equivalences involve the Taylor solutions, and their convergence locus. The Taylor solution of a differential equations over $\mathcal{E}_{K}$ does not converge anywhere.

However the computations we have obtained show that the solutions of differential equations and of $q$-difference equation over $\mathcal{E}_{K}$ coincide. Moreover by the canonical extension theorem for differential and $q$-difference equations one knows that, if $|q-1|<\omega$, then every rank one object comes by scalar extension from an object over the affinoid domain $A:=\mathbb{P}^{1}-\mathrm{D}^{-}(0,1)=\{|x| \geqslant 1\}$. In particular, for all $r>1$, every object comes by scalar extension from an object over the closed annulus $\{|x| \in[1, r]\}$. Hence we can apply the deformation and the confluence to the canonical extensions.

\section{REFERENCES}

ADV04 Yves André and Lucia Di Vizio, q-difference equations and p-adic local monodromy, Astérisque (2004), no. 296, 55-111. MR MR2135685

Chr81 Gilles Christol, Décomposition des matrices en facteurs singuliers. Applications aux équations différentielles, Study Group on Ultrametric Analysis. 7th-8th years: 1979-1981 (Paris, 1979/1981) (French), Secrétariat Math., Paris, 1981, pp. Exp. No. 5, 17. MR MR628158 (83h:12040)

Chr83 Modules différentiels et équations différentielles p-adiques, Queen's Papers in Pure and Applied Mathematics, vol. 66, Queen's University, Kingston, ON, 1983.

CM02 Gilles Christol and Zoghman Mebkhout, Équations différentielles p-adiques et coefficients p-adiques sur les courbes, Astérisque (2002), no. 279, 125-183, Cohomologies p-adiques et applications arithmétiques, II. MR 1922830 (2003i:12014)

CP09 Bruno Chiarellotto and Andrea Pulita, Arithmetic and differential Swan conductors of rank one representations with finite local monodromy, Amer. J. Math. 131 (2009), no. 6, 1743-1794. MR 2567506 (2011a:14036)

CR94 G. Christol and P. Robba, Équations différentielles p-adiques, Actualités Mathématiques., Hermann, Paris, 1994, Applications aux sommes exponentielles. [Applications to exponential sums].

DGS94 B. Dwork, G. Gerotto, and F. J. Sullivan, An introduction to G-functions, Annals of Mathematics Studies, vol. 133, Princeton University Press, Princeton, NJ, 1994. 
Solvability of RANK one $p$-ADIC Differential AND $q$-DIfFEREnCE EQUATIONS Over the Amice RING

DV04 Lucia Di Vizio, Introduction to p-adic q-difference equations, Geometric aspects of Dwork theory. Vol. I, II, Walter de Gruyter GmbH \& Co. KG, Berlin, 2004, pp. 615-675.

Fon90 J. M. Fontaine, Représentations p-adiques des corps locaux. I, The Grothendieck Festschrift, Vol. II, Progr. Math., vol. 87, Birkhäuser Boston, Boston, MA, 1990, pp. 249-309.

Kat87 Nicholas M. Katz, On the calculation of some differential Galois groups, Invent. Math. 87 (1987), no. 1, 13-61. MR MR862711 (88c:12010)

Ked10 Kiran S. Kedlaya, p-adic differential equations, Cambridge Studies in Advanced Mathematics, vol. 125, Cambridge Univ. Press, 2010.

Mat02 Shigeki Matsuda, Katz correspondence for quasi-unipotent overconvergent isocrystals, Compositio Math. 134 (2002), no. 1, 1-34. MR MR1931960 (2003j:12007)

Mot77 Elhanan Motzkin, La décomposition d'un élément analytique en facteurs singuliers, Ann. Inst. Fourier (Grenoble) 27 (1977), no. 1, ix, 67-82. MR MR0470247 (57 \#10005)

Pul07 Andrea Pulita, Rank one solvable p-adic differential equations and finite abelian characters via Lubin-Tate groups, Math. Ann. 337 (2007), no. 3, 489-555. MR MR2274542

Pul08 , p-adic confluence of q-difference equations, Compos. Math. 144 (2008), no. 4, 867-919. MR MR2441249 (2009f:12006)

Pul14 , Infinitesimal deformation of p-adic differential equations on Berkovich curves, arXiv:0802.1945v3 [math.NT] (2014), 39 pages.

Andrea Pulita pulita@math.univ-montp2.fr

Département de Mathématiques, Université de Montpellier II, CC051, Place Eugène Bataillon, F-34 095, Montpellier CEDEX 5. 